\title{
Binaries with the eyes of CTA
}

J.M. Paredes ${ }^{\mathrm{a}}$, W. Bednarek ${ }^{\mathrm{b}}$, P. Bordas ${ }^{\mathrm{c}}$, V. Bosch-Ramon ${ }^{\mathrm{d}}$, E. De Cea del Pozo ${ }^{\mathrm{e}}$, G. Dubus ${ }^{\mathrm{f}}$, S. Funk $^{\mathrm{g}}$, D. Hadasch $^{\mathrm{e}}$, D. Khangulyan ${ }^{\text {, }, ~ S . ~ M a r k o f f ~}{ }^{i}$, J. Moldón ${ }^{\mathrm{a}}$, P. Munar-Adrover ${ }^{\mathrm{a}}$, S. Nagataki ${ }^{\mathrm{j}}$, T. Naito ${ }^{\mathrm{k}}$, M. de Naurois ${ }^{1}$, G. Pedaletti ${ }^{\mathrm{e}}$, O. Reimer ${ }^{\mathrm{m}, \mathrm{n}}$, M. Ribóa ${ }^{\mathrm{a}}$, A. Szostek ${ }^{\mathrm{n}, \mathrm{o}}$, Y. Terada ${ }^{\mathrm{p}}$, D.F. Torres ${ }^{\mathrm{q}, \mathrm{e}}$, V. Zabalza ${ }^{\mathrm{a}, \mathrm{r}}$, A.A. Zdziarski ${ }^{\mathrm{s}}$, for the CTA Consortium

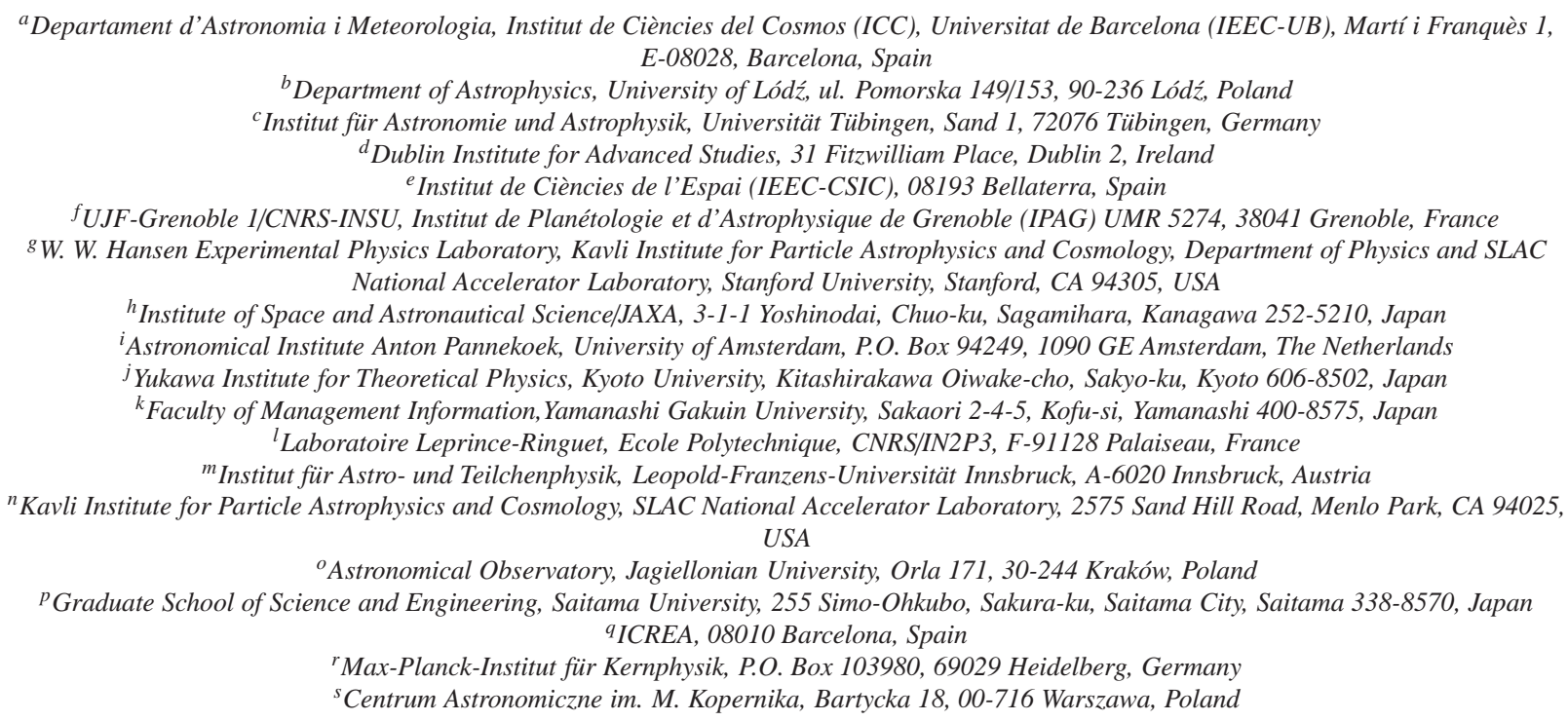

\begin{abstract}
The binary systems that have been detected in gamma rays have proven very useful to study high-energy processes, in particular particle acceleration, emission and radiation reprocessing, and the dynamics of the underlying magnetized flows. Binary systems, either detected or potential gamma-ray emitters, can be grouped in different subclasses depending on the nature of the binary components or the origin of the particle acceleration: the interaction of the winds of either a pulsar and a massive star or two massive stars; accretion onto a compact object and jet formation; and interaction of a relativistic outflow with the external medium. We evaluate the potentialities of an instrument like the Cherenkov telescope array (CTA) to study the non-thermal physics of gamma-ray binaries, which requires the observation of high-energy phenomena at different time and spatial scales. We analyze the capability of CTA, under different configurations, to probe the spectral, temporal and spatial behavior of gamma-ray binaries in the context of the known or expected physics of these sources. CTA will be able to probe with high spectral, temporal and spatial resolution the physical processes behind the gamma-ray emission in binaries, significantly increasing as well the number of known sources. This will allow the derivation of information on the particle acceleration and emission sites qualitatively better than what is currently available.
\end{abstract}

Keywords: Gamma-rays: observations - Binaries: general - Acceleration of particles - Cherenkov astronomy Radiation mechanisms: non-thermal - Telescopes

Email address: jmparedes@ub.edu (J.M. Paredes)

Preprint submitted to Astroparticle Physics

\section{Introduction}

The many spectacular discoveries made in recent years by both satellite-borne (AGILE, Fermi) and 
ground-based gamma-ray telescopes (H.E.S.S., MAGIC and VERITAS) have revealed a variety of new sources of high-energy particles in the Galaxy. Among these sources we can mention star-forming regions, accreting black holes and microquasars, early-type stars with very strong stellar winds, young isolated pulsars and their nebulae and pulsars in binary systems. The physics of particle acceleration and interaction in the complex environment of such astrophysical systems is extremely rich. The detection of very high energy (VHE) gamma rays $(E>100 \mathrm{GeV})$ by the current imaging atmospheric Cherenkov Telescopes (IACT) from the systems PSR B1259-63 [1, 2], LS 5039 [3], LS I +61 303 [4, 5] and HESS J0632+057 [6], as well as the hint of a VHE flare in the black hole binary Cygnus X-1 [7], provides a clear evidence of very efficient particle acceleration in binary systems containing compact objects (see e.g. [8]). In addition, one of the brightest Fermi/LAT sources, 1FGL J1018.6-5856, has been proposed to be a new gamma-ray binary [9] that could be associated with a H.E.S.S. source [10]. Furthermore, there are other binary systems from which VHE emission is expected from a theoretical point of view [11]. Although they have not yet been detected with the current generation of Cherenkov telescopes, their emission at HE gammarays $(E>100 \mathrm{MeV})$ has already been reported in some cases (e.g. Cygnus X-3, V 407 Cyg and Eta Carinae). It is expected that CTA will find new gamma-ray binaries, allowing population studies that will have an impact on evolutionary models of high-mass binary systems. With a few exceptions, most of the gamma-ray binaries detected, either accreting or non-accreting sources, are all within $3 \mathrm{kpc}$ of the Sun, in a volume equal to about $\sim 10 \%$ of the volume of our Galaxy. Assuming a uniform distribution, although they should follow population I stars with more objects in the spiral arms, this is consistent with $>50$ or so gamma-ray binaries in our Galaxy. This number is also dependent on the duty cycle of gamma-ray emission: VHE emission in HESS J0632+057, LS I +61 303, PSR B1259-63 is strongly dependent on orbital phase and in some sources the orbital periods can be (very) long. With a ten times improvement in sensitivity, CTA should be able to probe for gamma-ray binaries of comparable luminosities up to the Galactic center. CTA can thus be reasonably expected to detect a couple of dozen gamma-ray binaries. The VHE counterparts of LS 5039, HESS J0632+057 and (possibly) 1FGL 1018.6-5856 were discovered in the H.E.S.S. Galactic Plane survey. The ten times more sensitive Galactic Plane survey planned for CTA should thus enable many discoveries of such systems, which are otherwise very difficult to uncover by X-ray, optical or radio surveys. A survey of the central portion of the galactic plane is planned for the beginning of CTA operation (see [12]), which will pinpoint new gamma-ray binaries candidates.

The study of known and/or new compact binary systems at VHE is of primary importance because their complexity allows us to probe several physical processes that are still poorly understood. Some of these systems are extremely efficient accelerators that could shed new light, and eventually force a revision of, particle acceleration theory (see e.g. [13]). The particle injection and radiation emission mechanisms in binary systems vary periodically due to an eccentric orbit and/or interaction geometry changes. This may provide information on the location of the high energy particles, on the energy mechanism(s) powering relativistic outflows, on the nature of the accelerated particles, and on the physical conditions of the surrounding environment. The presence of strong photon fields allows the study of photon-photon absorption and electromagnetic cascades. All these processes occur on timescales $\lesssim 1000$ $\mathrm{s}$, a proper study of which would require at least a $5 \sigma$ (standard deviations) detection for $\sim$ one hour exposure times.

The interaction of binary systems with the Interstellar medium (ISM) could also be powering a new class of $\mathrm{TeV}$ sources, which could be resolved/detected with enough resolution/sensitivity. For a deep study of the processes taking place in compact binary systems we need to go beyond the present IACT's capabilities. Below, we report on examples of numerical simulations performed to show how the forthcoming CTA observatory [14] could fulfill these objectives.

The structure of the paper is as follows: in Sect. 2 we outline the stellar gamma-ray source classes that are idoneous targets for CTA. In Sect. 3 we introduce key questions in high-energy astrophysics that CTA can address and the requirements to achieve them. In Sect. 4 we present the results of some performance tests of the capability of CTA to achieve the aims. Finally we present a summary in Sect.5

\section{Binary systems with gamma-ray emission}

\subsection{Binary systems with young non-accreting pulsars}

PSR B1259-63 was the first variable galactic source of VHE gamma-rays discovered [1]. It has also been detected at HE by AGILE [15] and Fermi/LAT [16, 17]. The system contains a 09.5 Ve main sequence donor (LS 2883) and a $47.7 \mathrm{~ms}$ radio pulsar orbiting its companion every 3.4 years in a very eccentric orbit (see 
[18] and references therein). Particles are accelerated in the shock between the relativistic wind of the young non-accreting pulsar and the stellar wind of the massive companion star [19, 20, 21]. These particles, by inverse Compton (IC) up-scattering of stellar UV photons should produce VHE gamma rays. The strong wind-wind interactions may also produce extended synchrotron radio emission, as recently reported by [18].

The other binary systems that have been unambiguously detected at $\mathrm{TeV}$ energies, showing gamma-ray flux modulations coincident with their orbital periods, are LS 5039 with $P_{\text {orb }} \approx 3.9$ d [22] and LS I +61 303 with $P_{\text {orb }} \approx 26.5$ d [23]. Fermi has also detected emission modulated with the orbital period in both systems [24, 25]. Although the nature (black hole or neutron star) of the compact object in LS I +61 303 and LS 5039 has not yet been determined [26, 27], both systems present some similarities with PSR B1259-63. They show variable milli-arcsecond scale radio structure [28, 29, 30], similar to that found in PSR B1259-63. VLBA images of LS I +61 303 obtained during a full orbital cycle show a rotating elongated morphology [30], which may be consistent with a model based on the interaction between the relativistic wind of a young non-accreting pulsar and the wind of the stellar companion [20]. A similar behavior has been observed in LS 5039. This system was observed with the VLBA during five consecutive days showing an orbital morphological variability, displaying one sided and bipolar structures, but recovering the same morphology when observing at the same orbital phase [31]. The broadband emission from radio to VHE gamma-rays of the three sources is variable and periodic, peaking at $\mathrm{MeV}-\mathrm{GeV}$ energies.

For LS I +61 303 and LS 5039, the GeV and $\mathrm{TeV}$ emission are well anticorrelated. In particular, in LS 5039, the GeV emission peaking around the compact object superior conjunction/periastron, and the TeV radiation around inferior conjunction/apastron. The GeV and $\mathrm{TeV}$ spectra are also roughly anticorrelated, with the $\mathrm{GeV}$ emission getting harder for lower fluxes, and the $\mathrm{TeV}$ emission for higher ones (e.g., [22, 32]). In both sources, the behavior is more or less compatible with radiation produced by IC and moderate gammaray absorption, processes through which the changing geometry along the orbit induces a modulation in both flux at $\mathrm{TeV}$ and $\mathrm{GeV}$, and spectrum at $\mathrm{TeV}$ (and $\mathrm{GeV}$ if cascades were important). Additional effects like varying radiative and adiabatic losses could also affect spectra and fluxes [33]. We note that also IC $\mathrm{e}^{ \pm}$pair moderate cascading can be important [34, 35, 36]. However, the relatively low flux around $10 \mathrm{GeV}$ in both LS 5039 and LS I +61 303, below the extrapolation of data and model predictions, strongly indicates that the emitter, although of likely leptonic+IC nature, should be quite complex (and probably located in the periphery of the binary system for LS 5039).

The most recent addition to the selected group of gamma-ray binaries emitting up to very high energies is HESS J0632+057 [37]. The source was initially detected by the H.E.S.S. experiment [6], but the subsequent non detection by VERITAS excluded it as a steady gamma-ray emitter [38]. The gamma-ray variability was confirmed recently by VERITAS and MAGIC which reported an increase of gamma-ray flux during 2011 February 7-9 (see Refs. [39] and [40], respectively). The increase in $\mathrm{TeV}$ flux coincides with the time of a large X-ray peak, that could imply that the same population of electrons is producing the $\mathrm{X}$ ray and $\mathrm{TeV}$ emission [41], similarly to that found in LS I +61 303 [42] and LS 5039 [33]. The source shows an X-ray periodicity of $321 \pm 5 \mathrm{~d}$ which has been associated to its orbital period $[41,43]$. No X-ray pulsations have been detected so far [44]. In the radio band, VLBI observations reveal an extended and variable nonthermal radio source [45], with its position being compatible with the B0pe star MWC 148. Overall, these results are very similar to the multi-wavelength data obtained for the other gamma-ray binaries, although HESS J0632+057 displays a spectral energy distribution (SED) one order of magnitude fainter. This may provide new constraints on the luminosity distribution of this subclass of binary systems in our Galaxy.

A promising candidate to be a new VHE gamma-ray source associated to a binary system has emerged recently. 1FGL J1018.6-5856 was discovered by searching for periodicities of Fermi sources, and shows intensity and spectral modulation at $\mathrm{GeV}$ energies with a 16.6-day period [9]. Given the variability of the proposed X-ray and radio counterparts, and the spatial coincidence with an $\mathrm{O} 6 \mathrm{~V}((\mathrm{f}))$ star, these authors proposed this source as a new gamma-ray binary.

\subsection{Microquasars}

Microquasars (MQ) are X-ray binaries (XRB) with relativistic jets emitting non-thermal radio emission through synchrotron radiation. The gravitational energy released by accretion feeds the relativistic radio jets and powers the non-thermal emission. Two MQs, Cygnus X-3 [46, 47] and Cygnus X-1 [48], have been recently detected at HE gamma-rays and there is evidence of VHE gamma-ray emission associated with Cygnus X1 during a flare [49] but not with Cygnus X-3 [50]. The gamma-ray emission detected by Fermi from Cygnus $\mathrm{X}-3$ is modulated with the orbital period of the system 
and is correlated with the radio emission, which is associated with the relativistic jets. Other MQs, like SS 433 [51], GRS 1915-105 [52] or Sco X-1 [53], have also been observed at HE and VHE but they have not been detected yet. They show however synchrotron radio emission that probes their capability to accelerate particles up to relativistic energies, and their transient nature makes necessary to look at the sources at the right time to detect their high-energy radiation [54]. The presence of strong photon fields provided by the companion star may offer a good scenario for the production of gammarays through IC processes [55]. Furthermore, protonproton interactions could also take place in the collision between relativistic protons and the stellar wind [56]. However, photon-photon absorption may also take place in the inner regions of the system, and the VHE photons may be strongly attenuated [57, 34, 58].

It is noteworthy that, potentially, the non-thermal processes leading to radio and high-energy radiation in high-mass microquasars can be difficult to distinguish from those in young non-accreting pulsars. As pointed out, e.g. in [59] and [60], the complex and variable/periodic radio structures found in gamma-ray binaries, some with a compact object of unknown nature, may be also produced by jet disruption or gammaray absorption and secondary radio emission, respectively. In addition, the processes underlying or affecting the high-energy radiation, for instance in LS 5039 and LS I +61 303 (as described in Sect.2.1), would also take place in high-mass microquasars. Therefore, despite some differences in the non-thermal radiation are expected, they are likely to be more of quantitative than of qualitative nature, and only high-quality data may be able to allow the identification of the underlying engine.

\subsection{Collision of the outflow with the interstellar medium}

The termination regions of MQ jets can also generate non-thermal emission. The detection of VHE emission from those regions would represent a new type of gamma-ray emitter. In a scenario similar to that found in the Sedov-expansion phase of supernova remnants (SNRs), MQ jets propagating into the medium are eventually decelerated, developing forward/reverse shocks into the ISM/jet-ejection. In these shocks particles can undergo efficient acceleration and could produce gamma-ray emission through IC, relativistic Bremsstrahlung and proton-proton collision processes. The dynamics of these interactions (see e.g. [61]) and the possibility of the production of gamma-ray emission (62], [63]) have been recently addressed. The expected fluxes may depend on the MQ jet power, the source age, the external medium particle density profile, and the duty cycle if transient, and could reach values $F_{\mathrm{E}>10 \mathrm{GeV}} \sim$ few $\times 10^{-14} \mathrm{erg} \mathrm{cm}^{-2} \mathrm{~s}^{-1}$, which is a few times below the sensitivity level of the current IACTs. These sources may be therefore good targets for the improved capabilities of CTA.

Large-scale interactions are also expected in the case of binaries hosting a young pulsar, when mixed stellar and pulsar winds interact with the environment. The expected radiative outcome of this interaction is similar to that of microquasar jets [64].

\subsection{Colliding wind binaries}

Hot stars can generate strong winds and form colliding wind binary systems (CWB). Shocks are expected to form in massive star binaries, in the region where the winds from both stars collide. Non-thermal synchrotron emission from the colliding wind region in one source has been detected [65], which indicates the presence of highly relativistic electrons (see also [66]). These systems may also be embedded in dense photon fields where IC losses would be unavoidable, making CWBs potential high-energy emitters [67, 68].

An extreme example is the Eta Carinae system [69]. Gamma-ray emission has been theoretically predicted from this source (see e.g. [70, 71]) and the emission has been tentatively confirmed recently by the Fermi/LAT [72, 73] and AGILE [74] instruments. The predominant $\mathrm{GeV}$ emission of Eta Carinae, shown in the top panel of Fig. 11 seems to agree with what is expected from IC and/or neutral pion decay processes in such type of system. At VHE, Eta Carinae has not been detected so far [75]. The reported HE flux levels and the spectrum make however this source a good target for CTA, since it will provide a significantly improved sensitivity at energies in the range 30 to $100 \mathrm{GeV}$ as compared to present IACTs. MAGIC observations of WR 146 and WR 147 produced the first bounds on the high-energy emission from Wolf-Rayet binary systems [76].

\subsection{Cataclysmic binaries}

Regarding cataclysmic binary systems, variable highenergy gamma-ray emission has been recently detected from an optical nova of the symbiotic star V407 Cygni [77. According to the source SED shown in the bottom panel of Fig. 1, the extrapolated VHE gamma-ray fluxes are too low for current IACTs sensitivities, and even with its improved capabilities, CTA may require of relatively long exposure times to detect it. 

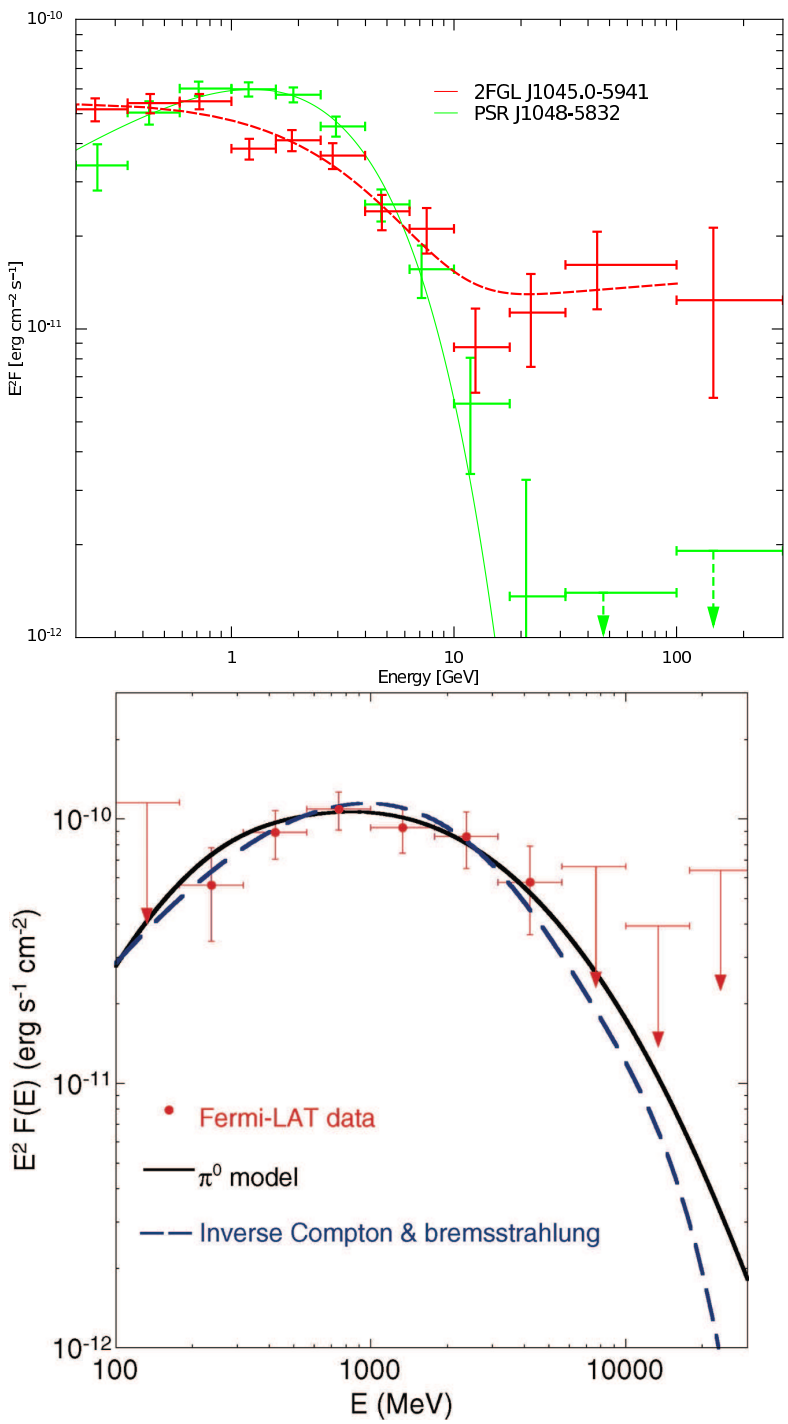

Figure 1: Top: Spectral energy distributions (SED) of two Fermi/LAT sources at the position of Eta Carinae: 2FGL J1045.0-5941 (red points) and PSR J1048-5832 (green points). The best-fit model for the average spectrum is shown as a red dashed line ([73]). Bottom: SED of V407 Cyg measured over the period from 10 March to 29 March 2010.

\section{Key questions and CTA requirements}

\subsection{Relativistic outflows in binary systems with young non-accreting pulsars}

The rapid rotation of the magnetosphere of PSR B1259-63 generates a highly relativistic wind that spins down the young neutron star. The properties of such winds are a long-standing issue of astrophysics. Constraints on pulsar winds have been inferred from observations of their interaction with the surrounding medium: the supernova ejecta in pulsar wind nebulae (PWN) or the ISM in isolated, fast moving pulsars. In a binary system, the pulsar wind interacts with the stellar wind of its companion in a periodic fashion. The binary nature determines the matter and radiation densities to be sampled by the pulsar during its orbital motion, and allows the production of relativistic outflows in the asymmetric postshock region of the pulsar wind. Phase-resolved spectra of binaries powered by a young pulsar offer novel tests of pulsar wind models on spatial scales $\sim 10^{4}-10^{5}$ times smaller than in isolated objects.

The study of pulsar winds requires phase-resolved light curves and spectra from binary pulsars. The systems LS 5039 and LS I +61 303 have been suggested to be powered by a young pulsar [20]. The orbital periods of these systems range from a few days (LS 5039) to years (PSR B1259-63). In LS 5039 the spectrum changes drastically between orbital phases of high flux as compared to phases of low flux. PSR B1259-63 and LS I +61 303, on the other hand, are only detected during high flux phases. Information on the low flux phases is therefore essential for modeling purposes, and this requires the increased sensitivity of CTA. An extended spectral range is also crucial to study the connection to the $\mathrm{MeV}-\mathrm{GeV}$ emission (due e.g. to IC emission from cascade electrons) and to constrain the high-energy cutoffs linked to particle acceleration.

Gamma-ray binaries, in particular LS 5039, present a very high acceleration efficiency $[13,33]$. CTA good sensitivity in the range $10-100 \mathrm{TeV}$ will allow us to probe the acceleration mechanism, the strength of the magnetic field, intrinsic orbital/short timescale variability of the accelerator/emitter, and the emitting particle nature. This will be possible since the opacity and orbital dependence of photon-photon absorption, and the angular IC dependence, sources of extrinsic variability, are smaller in this energy range. In addition, the spectrum of the emission is expected to be different depending on whether the emitting particles are protons or electrons.

Finally, several studies have tried to synthesize the population of high-mass X-ray binaries from assump- 
tions on the progenitor distributions and their evolutionary paths. Some include predictions on the number of binaries in the spinning down pulsar+massive star stage as a byproduct [78, 79, 80]. They are quite uncertain, with numbers ranging from 100 to 1000 depending on the details of binary evolution and on the adopted lifetime of this phase. Constraining the population of PSR B1259-63-like objects is important as this impacts HMXB populations but, ultimately, also the population of double neutron stars that are targeted by Virgo/LIGO.

\subsection{The accretion/ejection link in microquasars}

The possible detection of Cygnus X-1 by MAGIC may indicate that MQs are able to emit VHE gammarays. These systems, like active galactic nuclei (AGN) and gamma-ray bursts (GRBs), are powered by accretion. How and where the energy is released are still poorly understood. Part of the material is ejected at relativistic speeds and this is invariably associated with non-thermal emission. Importantly, emission from both accretion and ejection processes is detected in MQs. Their interplay can be studied when the mass accretion rate changes during outbursts. The MQ environment is better constrained and the timescales are more accessible than in GRBs and AGNs. Emission beyond a few $\mathrm{MeV}$ in some MQs found by CGRO/COMPTEL is essentially terra incognita, probably because of variability and lack of sensitivity. Exploring the VHE domain and connecting it to the known anti-correlations between thermal X-ray emission (from accreting material) and non-thermal radio emission (from the jet) should bring new insights into the accretion/ejection processes. Determining the time variability of the TeV to X-ray spectrum will help to know whether the jets have electronpositron or hadronic components, and will help as a consequence to understand the physics of the jet production. Interestingly, powerful radio flares are likely associated with the production of gamma rays [47, 81, 82]. As these radio flares occur in a specific area of the hardness intensity diagram, relatively precise predictions of a suitable trigger for CTA can be made.

Achieving this goal requires the monitoring of selected sources or target-of-opportunity (ToO) quick response with enough sensitivity to detect flaring episodes on $\sim$ hour timescales. The discrete ejections observed in X-rays, infrared and radio in GRS 1915+105, for instance, occur on such timescales, and these could also be detected in gamma rays. The full CTA array should detect a 10 -mCrab flare in one hour, corresponding to a luminosity above $100 \mathrm{GeV}$ of about $10^{-5} L_{\mathrm{Edd}}$ for a $10 \mathrm{M}_{\odot}$ black hole at the Galactic center distance. A significant advantage of CTA compared to current IACTs is subarray observations of specific sources. This will allow long-term monitoring that is currently too time-consuming for presently operating IACTs. A 0.1-Crab flare would be detected in one hour by a HESS-like subarray and could trigger follow-up observations of the whole array. Relating this emission to the known disk/jet/corona phenomenology will require on the one hand multi-wavelength campaigns, and on the other hand to have the possibility of a quick CTA response to ToO's from Fermi/LAT, X-ray satellites or radio interferometers like LOFAR and the SKA pathfinders MeerKAT, ASKAP and ATA. We note that ASKAP and LOFAR are starting all-sky transient monitoring in 2012.

\subsection{Collision of the outflow with the interstellar medium}

MQs are capable to transfer large amounts $\left(\sim 10^{49}-\right.$ $10^{51} \mathrm{erg}$ ) of kinetic energy and momentum to the surroundings through discrete or continuous ejections. Significant non-thermal gamma-ray emission in the jet/medium shocked regions could be produced, although dense ambient conditions are required. ISM particle densities can vary from $\sim 10^{-3} \mathrm{~cm}^{-3}$ (hot ISM regions) to $\sim 10^{4} \mathrm{~cm}^{-3}$ (inside molecular clouds). In the case of typical galactic densities $\sim 1 \mathrm{~cm}^{-3}$, the predicted VHE fluxes above $100 \mathrm{GeV}$ are a few $\times 10^{-14} \mathrm{erg} \mathrm{s}^{-1} \mathrm{~cm}^{-2}$, accounting for both relativistic Bremsstrahlung and $\mathrm{p}-\mathrm{p}$ interactions. The size of the interaction structures would be $\sim 1$ pc, i.e. $\sim 1$ arcminute for a source located at $3 \mathrm{kpc}$. The detection of VHE emission from the jet/medium interaction structures would reveal a new type of VHE emitter, probing the acceleration/radiation from large-scale shocks and constraining fundamental properties of galactic jets (e.g. energetics, magnetic field, etc.).

To detect the jet/medium interaction region requires to improve the present IACTs sensitivity $\left(\sim \mathrm{few} \times 10^{-13}\right.$ $\mathrm{TeV} \mathrm{s}^{-1} \mathrm{~cm}^{-2}$ ) about one order of magnitude and to have an angular resolution of 1 arc-minute. Additionally, the possibility of probing the $\lesssim 50 \mathrm{GeV}$ energy range would be extremely helpful, since this is where most of the gamma-ray flux is expected to be produced. There are no required special timing/monitoring CTA configurations, since the emission is expected to be steady and the source injection variability is supposed to be smoothed out at the large termination regions.

Similar requirements to those of jet termination regions are met also when observing the termination of outflows produced in pulsar binaries. 


\subsection{Colliding winds of massive stars in binary systems}

VHE emission is theoretically expected from binary systems with high-mass loss and high-velocity winds. These systems display some of the strongest sustained winds among Galactic objects and have the highest known mass-loss rate of any stellar type. Colliding winds of massive star binary systems are potential VHE gamma-ray emitters, via leptonic and/or hadronic processes after acceleration of primary particles in the collision shocks.

The detection of VHE emission from colliding winds requires an improved sensitivity with respect to current IACTs. To further study these systems in case of detection, phase-resolved light curves and spectra would be required. Such spectra could give us a clue to understanding the physical processes behind the emission, since the non-thermal particle distribution strongly depends on the shock conditions at each orbital phase. A low-energy array is favoured; a cutoff at a level of $\sim 100$ $\mathrm{GeV}$ due to the modest shock velocity and finite size of the acceleration zone is predicted, and CTA should be able to operate at a lower energy threshold than present IACTs. Finally, the orbital distances between the two stars $\left(\sim 10^{13}-10^{15} \mathrm{~cm}\right)$ make the emission region too small to be resolved out by the current designs of CTA configurations. Angular resolution is therefore not a requirement in this case.

\section{Performance tests}

Several of the studies herein presented are meant as examples of the capability of CTA in comparison with current IACTs; thus we take here several of the already detected gamma-ray binaries, for which a well known set of assumptions can be established. We have used Monte Carlo simulations of the sensitivity of the array for several possible configurations in order to explore the capability of CTA to study binary systems. In particular, we have conducted simulations using configurations $\mathrm{B}, \mathrm{D}, \mathrm{E}, \mathrm{I}, \mathrm{NA}$ and NB, and subarrays 1 s4-2-120, and s9-2-120. The southern configurations are optimized for low energies (B), high energies (D), balanced with a focus on low energies (E), and balanced with a focus on medium and high energies (I).

\footnotetext{
${ }^{1}$ The first number indicates the number of telescopes, the second indicates the type ( 1 for small size telescope, 2 for medium and 3 for large), and the last number indicates the separation in meters. The subarray s9-2-120 is composed of nine medium sized telescopes located at a distance of $120 \mathrm{~m}$ from each other.
}

The northern configurations are optimized for low energies (NA), and balanced (NB). The subarrays are HESSlike arrays (s4-2-120) and expanded HESS-like arrays (s9-2-120). All details can be found in Hinton et al., this issue.

\subsection{CTA flux error reduction in known TeV sources}

Accurate estimates of the flux, spectral shape, and evolution of known $\mathrm{TeV}$ sources are very important for constraining the physical parameters of the high-energy emitting region. This is even more needed when there are several parameters that have to be left free when fitting data. To explore the CTA capability to derive observables and constrain theoretical models, we simulated the CTA response on LS 5039. This source might not be representative of the class of binaries, but will allow us to compare the improvement from CTA data on the present generation of IACTs. We based our simulations on the results obtained by H.E.S.S. on the source, simulating the CTA response under similar conditions (above $1 \mathrm{TeV}$ ). Since the H.E.S.S. data were taken over a long time span and under different zenith angles, the energy threshold was not constant. To make a fair comparison with the H.E.S.S. analysis, we treated our simulated data the same way as the H.E.S.S. collaboration did [22]. The simulated counts and the flux normalization were extracted above $1 \mathrm{TeV}$ assuming an average photon index derived from all data: $\Gamma=2.23$ for $\mathrm{d} N / \mathrm{d} E \sim E^{-\Gamma}$. Based on the H.E.S.S. results we assumed a sinusoidal shape of the light curve with a period of $3.9 \mathrm{~d}$. For each phase point, and using configuration I, we then evaluated the light curve and simulated spectra of the form described above as seen by CTA, for a certain observation time in each phasogram bin. By integrating these spectra, we got the flux above $100 \mathrm{GeV}$ in each bin. We then extrapolated the obtained flux value to the integral flux above $1 \mathrm{TeV}$ and propagated the error correspondingly. The result for 70 hours of exposure time of CTA is shown in Figure 2 . The improvement of observations by CTA is clearly visible. The error bars are reduced by a factor of $\sim 2-4$ with respect to the H.E.S.S. data points, assuming the average photon index of $\Gamma=2.23$. This can be taken as a direct comparison to the published H.E.S.S. results. However, the spectral index of the LS 5039 VHE emission changes as a function of orbital phase. A variation of the spectral index affects the error estimate. Softening the assumed photon index $(\Gamma \sim 3)$, CTA data would improve the errors by up to a factor of 7 , whereas a harder spectrum $(\Gamma \sim 2)$ results in a minor error reduction of less than a factor of 2. We have also performed similar simulations but taking a time binning of 10, 20, 50 and 100 bins per 
full phase period and using a binning of 14 and 28 minutes. With 10 bins per full orbital period the sinusoidal shape can hardly be seen, whereas with 20 bins a sine function can be fitted to the data points. Taking 50 bins of 28 minutes even substructures can be resolved. To obtain the same results and similar error bars like those reported by H.E.S.S., CTA would only need $\sim 1 / 6$ th of its observation time, that is, 50 bins of $14 \mathrm{~min}$ each, pointing towards the possibility of performing a longterm monitoring of the global behavior of the source, and accessing the duty cycle of the observed features, if any.

Furthermore we studied the minimum observation time for CTA to detect the period of LS 5039 in comparison with the H.E.S.S. one. To do so, we simulated CTA observations using a sine function over time that reproduces the time structure of the H.E.S.S. flux points. From the simulated CTA observations we derived flux points for each time bin and used those to construct the power spectrum of LS 5039. Whereas H.E.S.S. used 160 bins of 28 minutes ( 70 hours in total) to detect the 3.9 days period of the system, CTA could detect the period with more than $5 \sigma$ with only 160 bins of $3 \min (8$ hours in total). This would be a significant reduction of observation time for CTA. It has to be kept in mind that the significance of the period estimation in the H.E.S.S. data is larger than $5 \sigma$ (i.e. $8 \sigma$ ), as all the data available at the time were used.

We studied the modulation of the photon index and the flux normalisation with the orbital period for a source like LS 5039. To compare with the H.E.S.S. measurements, we assumed 7 hours of observation time for each phase bin and simulated the CTA spectra for each phase bin with the spectral parameters obtained by H.E.S.S. (photon index and differential flux at $1 \mathrm{TeV}$ ). By fitting these simulated spectra, we obtained the fit parameters with the corresponding error. The results are shown in Figure 3 The direct comparison of the errors of the H.E.S.S. and CTA measurements shows that observations with CTA can reduce the errors on the spectral parameters by a factor between 2 and 4.5 .

The larger sensitivity of CTA would allow tracking the behavior of a source in shorter timescales. In particular, it would allow comparing with predictions of the spectral evolution of a source such as LS 5039, even at the minimum of its $\mathrm{TeV}$ flux. As an example, we used the spectra in phases 0.2 and 0.3 as derived by [83], where electromagnetic cascades were included. In Figure 4 we show the results of our simulations: in the top panel, the two simulated spectra are plotted, assuming an observation time of 5 hours. Since the reconstruction of the energy spectra in true energy requires

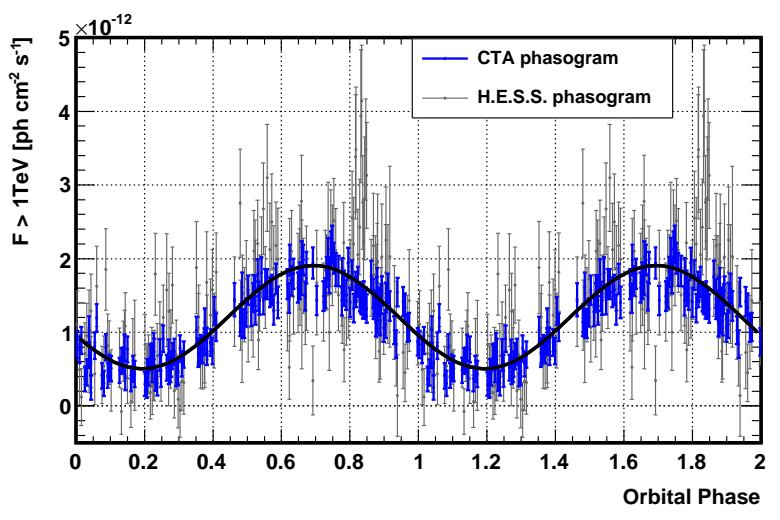

Figure 2: CTA simulations of LS 5039. Phasograms of H.E.S.S. (gray) and simulated CTA observations (blue). With CTA observations the error on the flux can be reduced by a factor $2-4$ above $1 \mathrm{TeV}$.
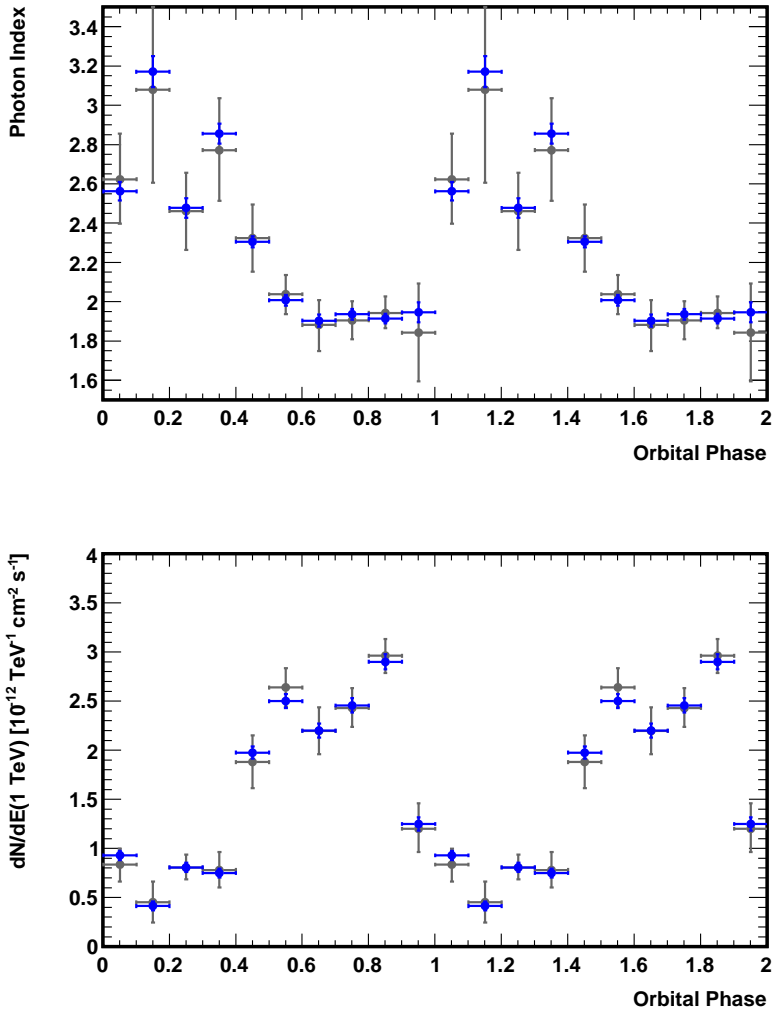

Figure 3: CTA simulations of LS 5039. Top: Photon index versus phase (CTA blue, H.E.S.S. gray). Bottom: Flux normalisation versus phase (CTA blue, H.E.S.S. gray). 

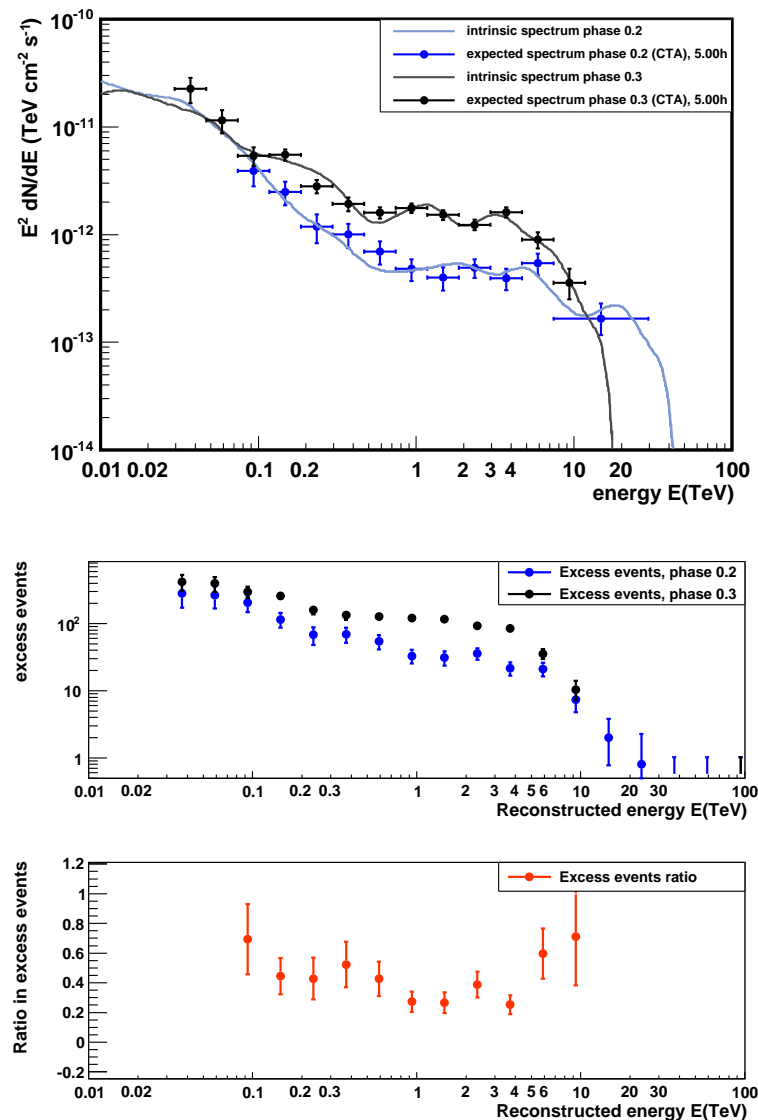

Figure 4: CTA simulations of LS 5039. Top: Intrinsic (line) and simulated (data points) energy spectra for phases 0.2 (blue) and 0.3 (black). The assumed integration time is $5 \mathrm{~h}$. Middle: Excess events obtained from the simulations above versus reconstructed energy. Bottom: Residuals of the excess events distributions from the two distributions above. In all three panels a very clear difference between the two spectra can be seen.

a complicated unfolding procedure we conservatively choose to compare the two spectra on the level of the excess events as a function of reconstructed energy. The two corresponding distributions are shown in the middle panel of Figure 4 The distributions are compared to each other by calculating the residuals between the two, which is shown in the bottom panel. The probability of these spectra to be consistent (i.e. to originate from the same original distribution) is $\ll 1 \%$. We conclude that CTA would easily distinguish between spectra at different phase bins.

\subsection{Short timescale flux variability}

We have studied the capabilities of CTA to detect short timescale flux and spectral variabilities from gamma-ray binaries. The perfect candidate for such studies is LS I +61 303. A simultaneous multiwavelength campaign on this source resulted in the discovery of correlated X-ray/VHE emission with the MAGIC IACT and the X-ray satellites XMM-Newton and Swift in the energy range $0.3-10 \mathrm{keV}$ [42] (see however [84, 85]). Additionally, fast X-ray variability on timescales of a thousand seconds has been detected [86, 87], so it is natural to think that such fast variability may be present in its VHE emission. The detection of such a variability would have strong implications on the location and size of the non-thermal emitter. Several theoretical models put forward until now consider the possibility of short timescale ( $100 \mathrm{~s})$, correlated X-ray/VHE variability as the result of leptonic emission in small scale instabilities and shocks within, e.g. a relativistic jet [88] or the interaction of the pulsar wind with a clumpy stellar wind [89]. The detection of such a feature with CTA will provide information on the dynamical mechanisms underlying the VHE emission, which is a crucial step in understanding the apparently erratic variability of LS I +61 303. This knowledge will be also valuable in understanding other gamma-ray binaries with complex stellar wind geometries owing to the decretion disk of Be stars, such as PSR B1259-63 and HESS J0632+057.

Under the assumption that the X-ray/VHE emission correlation found by [42] holds at shorter timescales, we took the X-ray light curve from a $100 \mathrm{ks}$ long Chandra observation [86] and computed the expected VHE emission from the source. We considered the large statistical errors in the correlation by varying the parameters following normal distributions with a width equivalent to the uncertainties for each of the points in the light curve.

We considered two feasible observation scenarios. In the first, the full CTA I array is used to perform a deep, short observation of LS I +61 303. In this case, the observation length would be of the order of $4 \mathrm{~h}$, which is the typical time span for which a source is visible under optimum conditions for a single night. To simulate this scenario, we selected $10 \mathrm{ks}$ of the X-ray observation and obtained the simulated VHE fluxes for energies above $100 \mathrm{GeV}$, taking advantage of the improved low-energy threshold of CTA with respect to MAGIC. We note that the CTA performance simulation is for an observation at low zenith angles, and a $4 \mathrm{~h}$ observation is likely to cover a large range of zenith angles resulting in an increased energy threshold.

CTA is able to clearly detect the fast variability exhibited by the X-ray light curve at scales down to $1000 \mathrm{~s}$, as well as recover the X-ray/VHE correlation, with $r \geq$ 0.9 , for flux variations of a factor 1.5. 

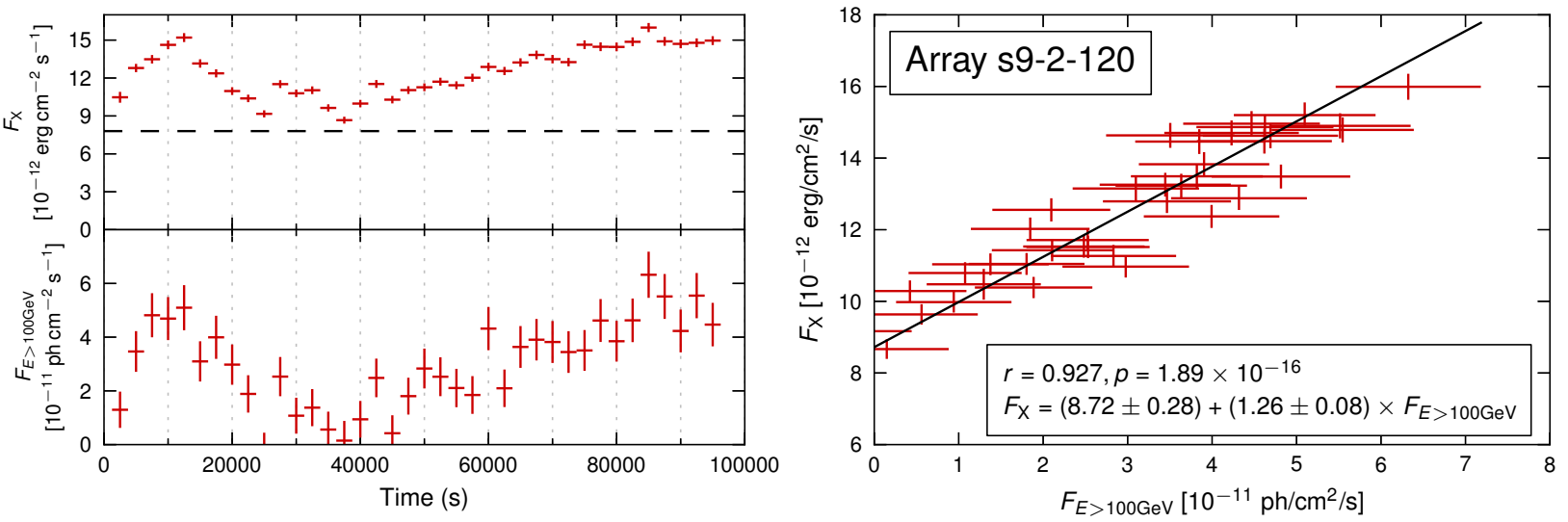

Figure 5: CTA simulations of LS I +61 303 during $100 \mathrm{ks}$, a time span similar to that of a 10 night observation campaign with around three hour of observation per night. Left: X-ray and VHE light curves for CTA s9-2-120. Measured X-ray flux (top, $0.3-10 \mathrm{keV}$ ) and simulated VHE fluxes obtained with the subarray s9-2-120 (bottom, $E>100 \mathrm{GeV}$ in units of $\mathrm{ph} \mathrm{cm}^{-2} \mathrm{~s}^{-1}$ ) for the case of a campaign of ten nights of observation and binning time of $2500 \mathrm{~s}$. Right: X-ray/VHE correlation for CTA s9-2-120. X-ray/VHE correlation plot from the ten-night, subarray s9-2-120 observation plotted in $2500 \mathrm{~s}$ bins. The Pearson correlation factor, the chance probability of correlation and the parameters of the fitted correlation are shown as insets.
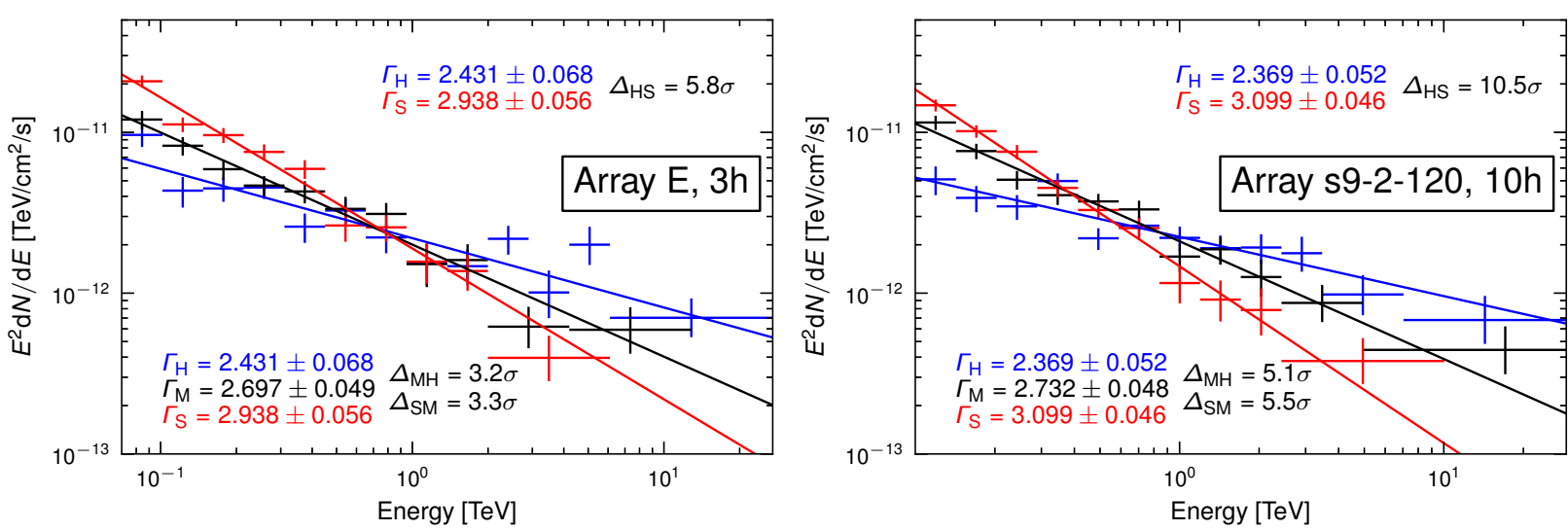

Figure 6: CTA simulations of LS I +61 303. Left: Sensitivity of CTA E to spectral variations. Simulated spectra and power-law fits for 3 hours of observation with the full CTA E array for each of the spectral states. The measured power-law indices and the significance of the variations are shown. Right: Sensitivity of CTA s9-2-120 to spectral variations. Simulated spectra and power-law fits for 10 hours of observation with the subarray s9-2-120 for each of the spectral states. The measured power-law indices and the significance of the variations are shown. 
The second observation scenario consists of a longer observational campaign of about ten nights with three hours of observation per night, which we here simulate from the continuous $100 \mathrm{ks}$ X-ray light curve. Given the foreseeable pressure to obtain observation time with CTA, this campaign would not be done with the full array but with a so-called subarray, a subset of the 100 telescopes that may operate independently. Here we chose a subarray configuration similar to an expanded H.E.S.S.: nine medium sized telescopes located at a distance of $120 \mathrm{~m}$ from each other, known as s9-2-120. This kind of configurations will be readily available after the construction of CTA begins, and thus the science case described could be achieved even before the whole array becomes operational. In Figure 5 (left) we see that the longer observation time allows the campaign to probe a wider range of X-ray fluxes than the variation of a factor 1.5 used for the shorter, full CTA array observation discussed above. Even with the reduced sensitivity of the subarray as compared to the full array, the correlation is well recovered (see Fig. 5 right), partly owing to the aforementioned wider X-ray flux coverage.

To further explore the shortest time scales in which CTA can resolve a flare, we simulated a 20 hours event whose flux variation follows a Gaussian distribution and assuming the best-fit spectral shape reported by MAGIC for the Cygnus X-1 signal [49]. 20 hours represent the total duration of the flare (i.e. the mean of the Gaussian distribution is at 10 hours from the start of the observation). With a binning of $5 \mathrm{~min}$ for each data point, CTA could clearly resolve this assumed Gaussian-shaped flare. A 5 min integration would result in a detection with a significance of $7 \sigma$ at the assumed low state and $25 \sigma$ in high state, whereas with the sensitivity of MAGIC it is only possible to detect the peak of this flare. This is a clear example of the better sensitivity of CTA with respect to the existing IACTs. Although the limited duty cycle of Cherenkov telescopes prevents them to observe a source for 20 hours in a row, a realistic exposure time of 5 hours would be enough for CTA to resolve parts of the flare in bins of $\sim 10 \mathrm{~min}$.

As a conclusion from this simulation exercise, we see that the full CTA array will be a powerful tool to probe into the fast flux variability of gamma-ray binaries, which could allow the characterization of the dynamical processes taking place in the emitting plasma. In addition, we have shown that subarray operations must be encouraged since they are able to provide very interesting results using only a fraction of the full array. They will prove invaluable not only in observational campaigns where the long-term variability is important, but also to monitor flaring sources such as gamma-ray binaries and AGNs.

\subsection{Sensitivity to spectral shape variations}

There have been some hints of VHE spectral variability of LS I +61 303 along the orbit [90], but they were not significant enough to be claimed as real. The proper characterization of the spectral variability would allow the characterization of the radiation mechanism, and more generally, as mentioned in Section 4.1, the determination of the conditions in the high-energy emitter. To explore the power of CTA to disentangle different spectral indices, we have considered a variation of the power-law VHE spectrum of LS I +61 303 within the statistical error obtained in the MAGIC campaign on September 2007 (See [42]) and test the time required by CTA to significantly detect it. The measured spectral index was $\Gamma=2.7 \pm 0.3$, so we took the two extreme values (2.4 and 3.0) and obtained the simulated CTA spectra. We computed the CTA spectra above the energy thresholds of $70 \mathrm{GeV}$ and $100 \mathrm{GeV}$ for the full array configuration $\mathrm{E}$ and the subarray s9-2-120, respectively. We found that an observation with the full CTA E array of 3 hours for each of the spectral states (i.e., one night per state) provides a set of spectra from which the photon index variations can be clearly seen. An example of such a realization can be seen in Fig. 6, left. The difference between the hard $\left(\Gamma_{\mathrm{H}}=2.4\right)$ and soft $\left(\Gamma_{\mathrm{S}}=3.0\right)$ is detected at a confidence level of more than $3 \sigma$ in $99 \%$ of the realizations, with a mean value of $(7.8 \pm 1.8) \sigma$. Considering the variation with the mean spectral shape of $\Gamma_{M}=2.7$, the significance of the variation with respect to the hard and soft spectra is lower but still above $3 \sigma$ for $85 \%$ and $74 \%$ of the realizations, respectively. We note that an increase in observation time to 5 hours per spectrum leads to a detection rate above $95 \%$ for all the spectrum pairs (see Figure 7 ). We also considered the possibility of longer observations with the subarray s9-2-120. In this case, for an observation of 10 hours for each of the spectral states, the spectral variation between the soft and mean spectra is still significant at a level higher than $3 \sigma$ for $82 \%$ of the simulation realizations. A representative realization of three simulated spectra with the subarray s9-2-120 can be seen in Fig. 6, right.

To further test the CTA spectral capabilities, we have used the derived spectrum of Cygnus X-1 during the flare to simulate 20 energy spectra with photon indices ranging from -2 to -4 . We have also simulated different exposure times: 5, 15, 30 and $60 \mathrm{~min}$, to study the minimum time scale to distinguish between the slope of different spectra. Figure 8 left shows the photon index error versus the simulated photon index in the fitting of 

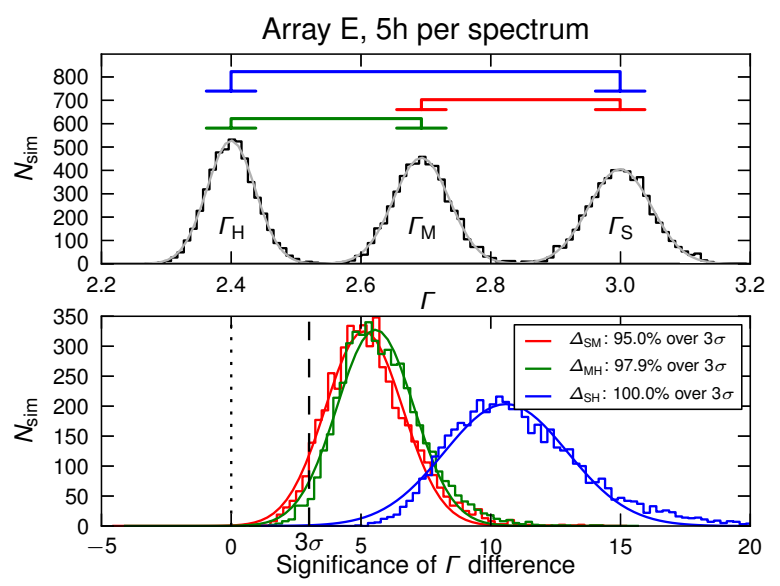

Figure 7: Results of 10000 realizations of the spectral observation of LS I +61 303 with the array E during $5 \mathrm{~h}$ per spectral state.Top: Distribution of recovered spectral indices for soft $\left(\Gamma_{\mathrm{S}}\right)$, mean $\left(\Gamma_{\mathrm{M}}\right)$, and hard $\left(\Gamma_{\mathrm{H}}\right)$ spectra. Bottom: Distribution of significances of the difference between each of the spectrum pairs. The legend indicates the fraction of realizations with a significance larger than $3 \sigma$.

the resulting CTA spectra. CTA would be able to distinguish the different spectral slopes in all cases except those showing the softest spectra, where the error bars are too large to properly distinguish them at a high confidence level. In that case, the observation of a flare as that reported in Cygnus X-1 would require exposures $\geq$ 15 min. Should such a kind of flare happen again, the minimum timescale for a 5 standard deviations detection of a flare within $10 \%$ of the reported spectrum from Cygnus X-1 is in the range of 2-3 min. However, in order to have a spectrum determination, one can consider a $10 \sigma$ detection threshold. With this constraint, the minimum timescale accessible is in the range of 8.5-12.5 min. This is shown in Fig 8 right. The above estimates hold, provided that the responses of the array are as stable as simulated for 30 minutes exposure and that the timescales are probed a priori.

It is clear that CTA will be a powerful tool for the detection of spectral variations in gamma-ray binaries. The statistical errors we obtained for these simulations are nearly an order of magnitude lower than the ones obtained with MAGIC in 2007, thus demonstrating the capability of CTA to deliver new and exciting science in the following years.

\subsection{Exploring the minimum detectable time delay be- tween $X$-ray and TeV emission in gamma-ray bina- ries}

As stated in section 4.2, the MAGIC collaboration reported on the existence of a correlation between the
X-ray emission and VHE gamma-ray emission from the gamma-ray binary LS I +61 303 [42] (see however [84]). The electron cooling times owing to synchrotron, IC and adiabatic losses inside the system are expected to be around a few thousand seconds (see, e.g. [91]). If there is a time delay between the non-thermal emission at different bands (e.g., X-ray and TeV) larger than the electron cooling time scale, the emission from these bands would most likely have an origin in different locations in the binary. The detection of such a delay has been impossible with the current generation of IACTs, so here we present a study of the capability of CTA for such a study. Furthermore, since CTA will operate together with a new generation of X-ray telescopes, such as the Japanese X-ray telescope Astro- $H$, we have done this study considering the capabilities of this new instrument. LS I +61 303 is the binary system selected for this study.

Based on the short X-ray flares from LS I +61 303 detected in [86], we have chosen to model a Gaussian flare with a width of $1500 \mathrm{~s}$. In the Chandra observations, these flares have a baseline count rate of $0.35 \mathrm{~s}^{-1}$ and a peak of $0.8 \mathrm{~s}^{-1}$, corresponding to unabsorbed energy fluxes of $\sim 8$ and $\sim 19$ times $10^{-12} \mathrm{erg} \mathrm{cm}^{-2} \mathrm{~s}^{-1}$, respectively. We used these parameters to simulate the light curve detected by Astro- $H$. Using the correlation between X-rays and VHE gamma rays found in [42] and the tools for simulating the CTA response, we generate the corresponding VHE light curves of the flare as seen by CTA array above $65 \mathrm{GeV}$ in configuration I. We have used a time binning of $600 \mathrm{~s}$ for both the CTA and the Astro- $H$ light curves (LC), and studied positive delays of the TeV light curve with respect to the X-ray light curve in the range 0 to $2000 \mathrm{~s}$ in steps of $100 \mathrm{~s}$. We show in the top panels of Figure 9 the simulated light curves corresponding to 500-s delay (left) and 1000-s delay (right). In the middle panels of Figure 9 we show the X-ray fluxes as a function of the $\mathrm{TeV}$ fluxes in both cases. A low correlation coefficient is obtained due to a loop structure induced by the delay. When the delay is 0 the average correlation coefficient is $r=0.86 \pm 0.04$.

To clearly detect such delayed correlations, we have used the $z$-transformed discrete correlation function (ZDCF), which determines $68 \%$ confidence level intervals for the correlation coefficient for running values of the delay (see, e.g. [92, 93]). We show in the bottom panels of Figure 9 the ZDCF in the case of 500-s delay (left) and 1000-s delay (right). The detection of correlated signals is clear in both cases. Since the VHE LC is generated from the X-ray one, their errors are correlated and the scattering of one original (X-ray) light curve affects the second one (VHE). To correct this problem we 

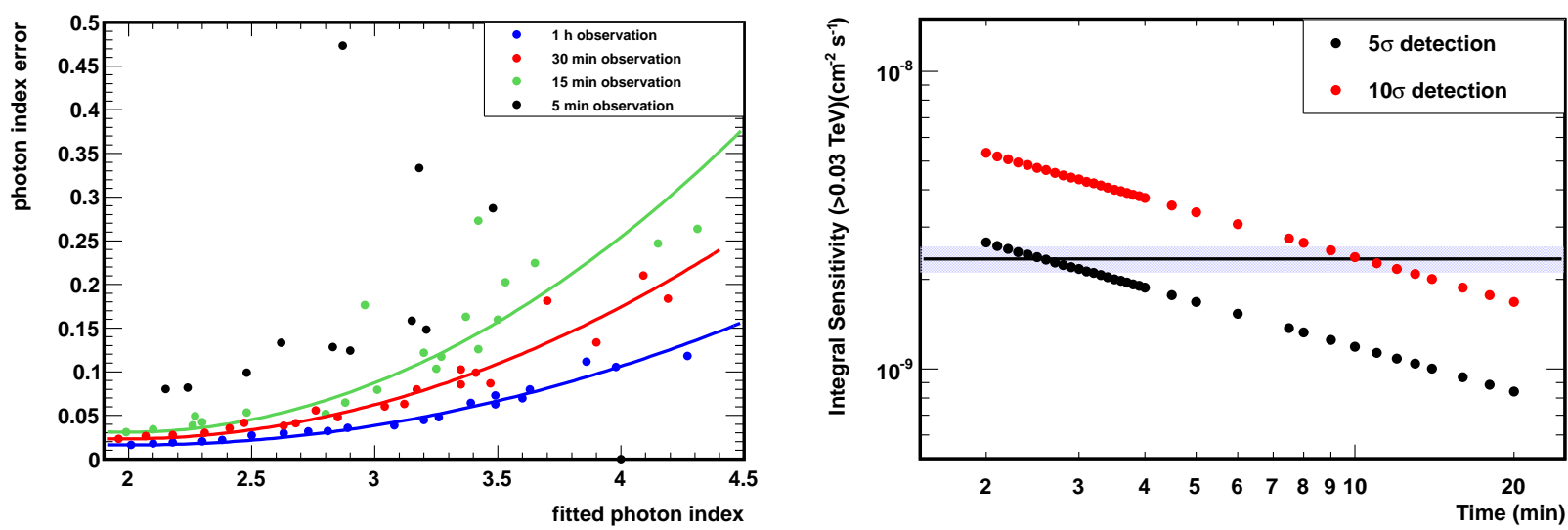

Figure 8: CTA simulations of Cygnus X-1. Left: Error on the photon index versus the fitted photon index. The photon index error can be understood as a proxy of the finest possible resolution. The lines show the fit of a parabolic function to the data points normalized to the first point. Right: Integral sensitivity above $30 \mathrm{GeV}$ as a function of integration time for the spectrum of Cygnus X-1 [7], above 5 and 10 standard deviations threshold (see text). The shaded area represents a variation of $10 \%$ from the spectrum of Cygnus X-1 during the 2007 flare.

went through simulations: we started with 100 Astro$H$ LC. With each of this $100 \mathrm{LC}$ we produced 10 other $\mathrm{X}$-ray LC by adding a Gaussian noise to the original ones, for each simulated delay. Then we simulated the corresponding VHE LC. At the end we had, for each delay, a sample of 1000 pairs of LC. For each pair of light curves we have calculated the ZDCF. To evaluate how significant is the measurement of a delay using the ZDCF we have fitted Gaussian functions to the maxima of the ZDCF. At the end we have 1000 values of the peak for each simulated delay distributed around the real delay (see Figure 10). The measured delay is then calculated as the mean value of the distribution and its uncertainty is the standard deviation. In Figure 11 we show the measured delay as a function of the simulated delay following the procedure described above. Considering all possible uncertainties, delays of $\sim 1000 \mathrm{~s}$ can be significantly detected at a $3 \sigma$ confidence level in simultaneous light curves obtained with Astro- $H$ and CTA. These results are, to first order, independent of the duration of the short flares, as far as they last longer than the binning. Overall, these results indicate that CTA will allow us to localize and constrain the $\mathrm{X}$-ray ant $\mathrm{TeV}$ emitting regions of gamma-ray binaries and their properties.

\subsection{Exploring the collision of microquasar jets with the interstellar medium}

To simulate the CTA response to the observation of MQ jet/ISM interactions, we have used the theoretical predictions for a source with a jet power of $10^{38} \mathrm{erg} \mathrm{s}^{-1}$, a source age of $10^{5} \mathrm{yr}$ embedded in a medium with particle density of $1 \mathrm{~cm}^{-3}$ [62]. Gamma-ray spectra with

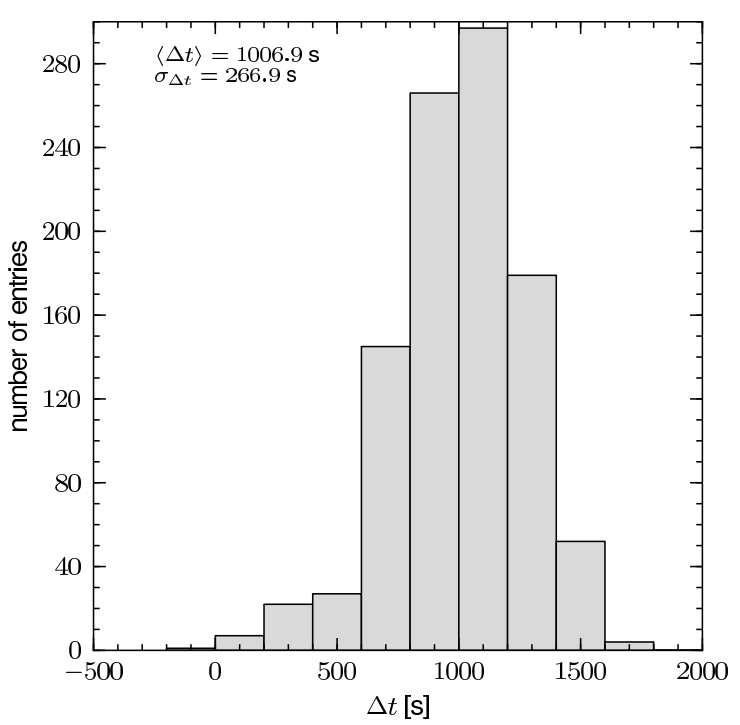

Figure 10: Distribution of the fitted peak of the ZDCF for the simulated light curves for an introduced delay of $1000 \mathrm{~s}$. A Gaussian fit gives the measured delay $\langle\Delta t\rangle$ and its standard deviation $\sigma_{\Delta t}$. 

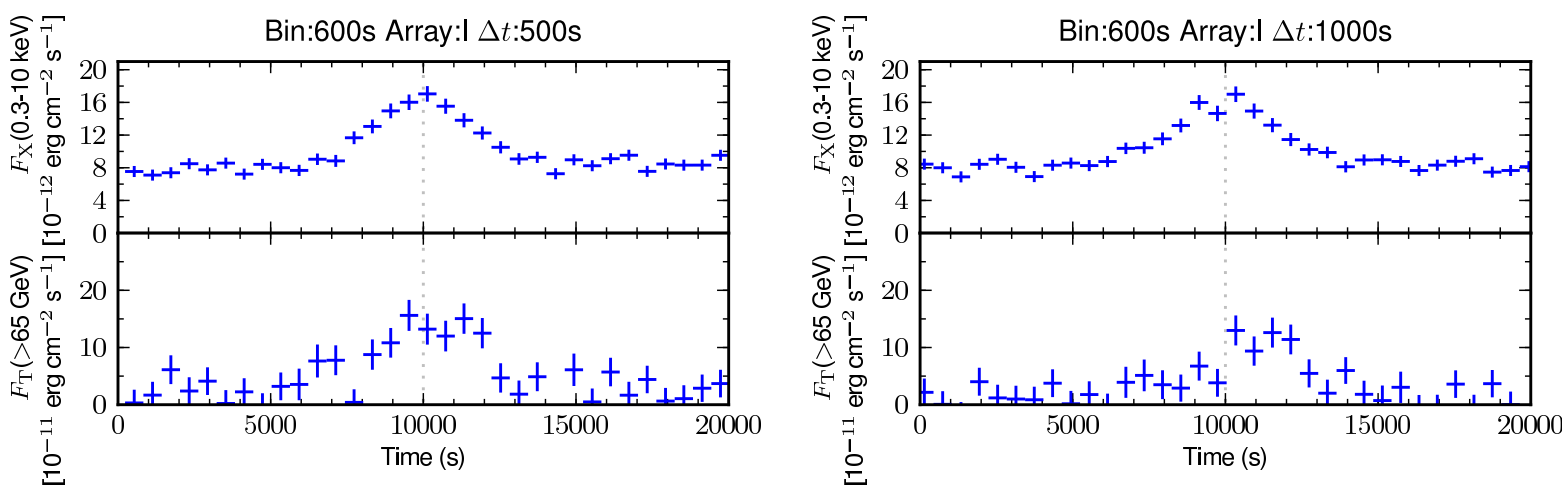

Bin:600s Array:I $\Delta t: 500$ s

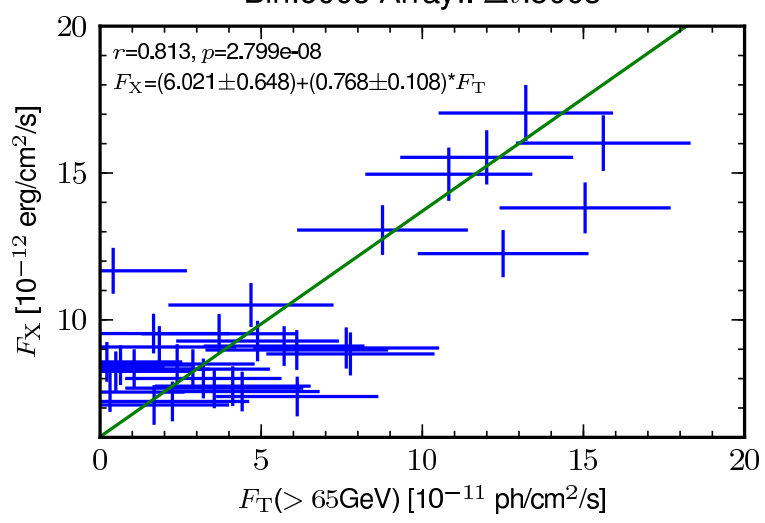

Bin:600s Array:I $\Delta t: 1000$ s
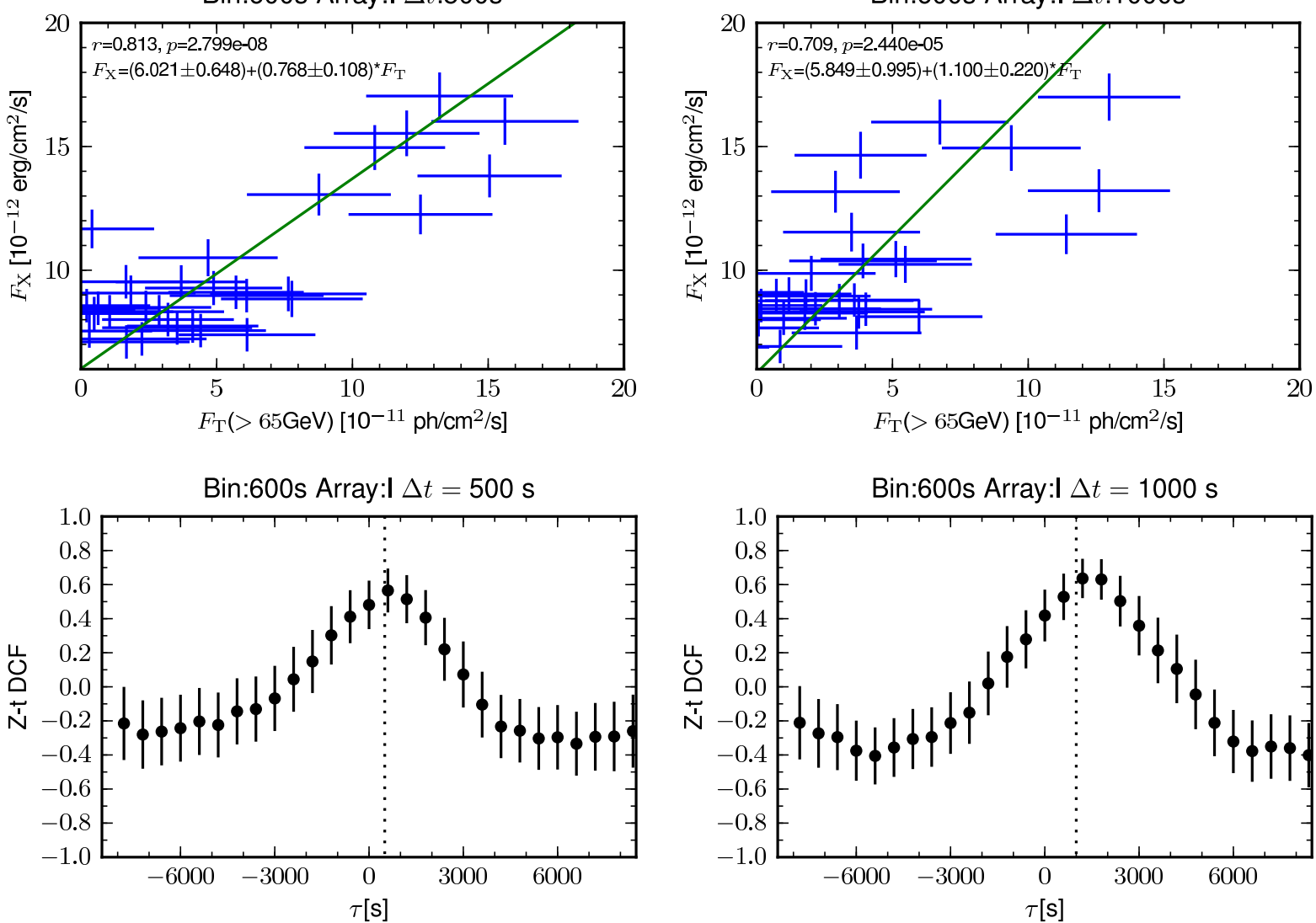

Figure 9: CTA simulations of LS I +61 303. Top left:X-ray and VHE light curves of a Gaussian flare with sigma of $1500 \mathrm{~s}$ and 500-s delay. Top right: same with 1000-s delay. Middle left: X-ray flux vs. VHE flux for 500-s delay. Middle right: same with 1000-s delay. Bottom left: ZDCF for 500-s delay. Bottom right: same with 1000-s delay. 


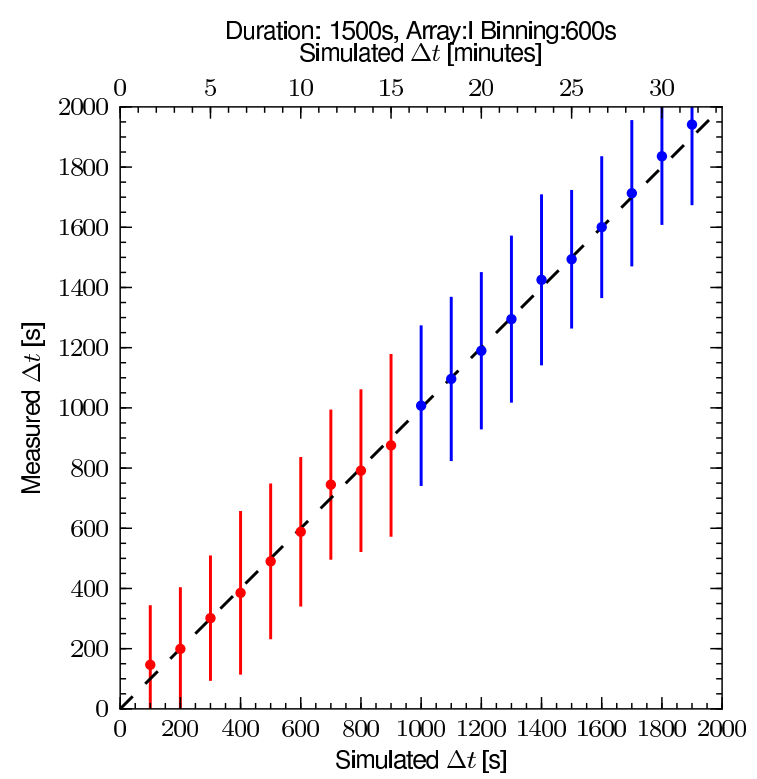

Figure 11: CTA simulations of LS I +61 303. Measured time delay as a function of the simulated delay. Delays as short as $1000 \mathrm{~s}$ can be significantly measured.

$\Gamma_{\mathrm{ph}} \sim 2.45$ and $\Gamma_{\mathrm{ph}} \sim 2.85$ are derived from current theoretical models for the leptonic and hadronic contribution, respectively [62, 63]. We studied the CTA performance in $50 \mathrm{~h}$ of observation time using the simulation tools for the B and E array configurations. Figure 12 shows the obtained SED. The simulated flux is at a level $\sim 1 \%$ of that of the Crab Nebula, although the steepening of the spectrum at high energies would make it difficult to detect the sources above a few TeV.

Regarding the extension of the emission as seen in gamma rays, accelerated particles emitting at VHE do not have time to propagate to large distances since radiative cooling is very effective. The emission will be mostly confined to the accelerator region itself. The emitter size may not largely exceed the width of the jet, $\sim 1 \mathrm{pc}$, in the reverse shock region. In the case of the bow shock, although it may extend sideways for much larger distances, only the region around its apex $(\sim$ few pc) will effectively accelerate particles up to the highest energies. The total angular size of the emission from a source located $3 \mathrm{kpc}$ away may then be $\lesssim$ few arcmin, and CTA would image a point-like source with only a marginal extension roughly perpendicular to the jet direction.

To resolve the $\mathrm{TeV}$ emission produced in the jet/medium interaction regions and disentangle it from the putative contribution produced close to the jet base, the reverse/bow shocks need to be located at a distance $\gtrsim 10^{19} \mathrm{~cm}$. Although the precise location of the interaction regions for a particular source can be difficult to predict since they depend on the jet power and age and the medium density surrounding the system, hot spots displaying non-thermal emission at similar distances have been found, e.g. in SS 433 [94]. Upper limits to the $\mathrm{TeV}$ emission from jet/medium interaction regions have been already reported for some microquasars, e.g. Cygnus X-1 49] and GRS 1915+105 [52]. However, clear evidences of non-thermal emission at the interaction sites in those cases are still lacking (see, e.g. [95, 96, 97]).

Steady gamma-ray fluxes are predicted in jet termination regions. A constant flux pedestal level could be observed on top of the orbital modulations expected from the central parts of the system for a wide range of orbital geometries and system inclinations due to IC and pair-creation angle dependencies in their respective cross-sections, and/or due to orbital variations of the accretion rate in both hadronic and leptonic scenarios. We note that CTA may not be able to separate the possible contribution coming from the reverse shock and the forward (bow) shocks. The shocked jet and medium material are separated by the contact discontinuity, and although the extent and relative position of it with respect to both shocks is difficult to predict (see e.g. [98]), it may be too short for the angular resolution at the level of few arcminutes expected for CTA. Possible fast diffusion of accelerated particles behind the shocks would complicate further the situation.

\subsection{Exploring the colliding winds of massive star bi- nary systems}

We performed numerical simulations of the response of CTA for a CWB like Eta Carinae. We based our simulations on the measurements of the energy spectrum of Eta Carinae (see top panel of Fig. 1) by the Fermi/LAT [73] and the upper limits derived by the H.E.S.S. Collaboration [99]. The spectrum between 0.1 and $100 \mathrm{GeV}$ is best fit by a power law with an exponential cutoff plus an additional power law at high energies. In the $\mathrm{TeV}$ range, Eta Carinae has not been detected. In Figure 13 (left) we show the Fermi/LAT data points and the H.E.S.S. upper limits in gray. From these measurements, it seems that there must be a cutoff in the spectrum at high energies. For our simulations we assume exponential cutoffs at $E=100,150,200 \mathrm{GeV}$ and test how well CTA could detect those. We produced simulations at increasing observation times in order to study the minimal time required to detect the source 


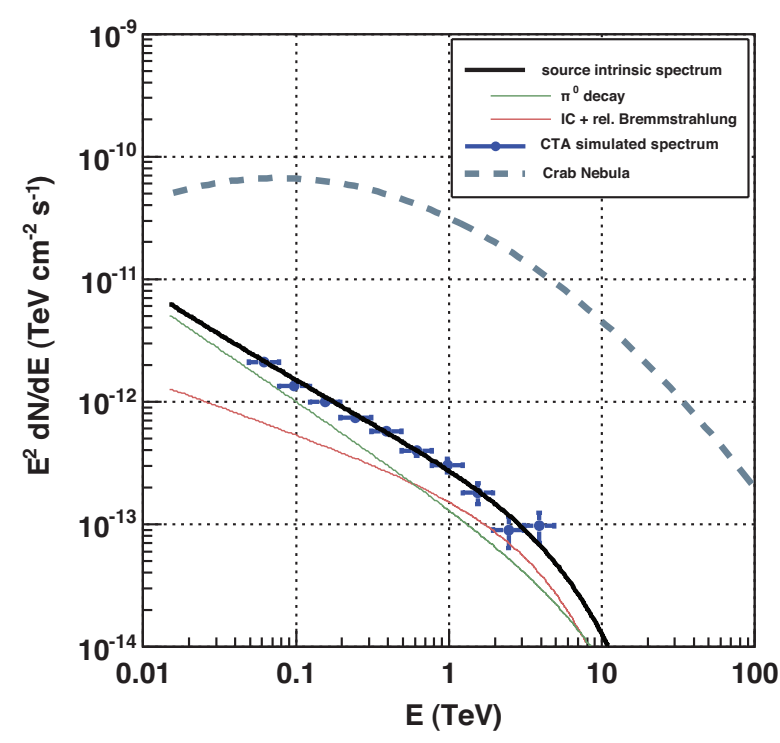

Figure 12: CTA simulations of the emission from the MQ jet/ISM interaction regions. A jet power of $10^{38} \mathrm{erg} \mathrm{s}^{-1}$, a source age of $10^{5} \mathrm{yr}$ and a medium with a particle density of $1 \mathrm{~cm}^{-3}$ have been used. The gamma-ray spectra of IC and relativistic Bremsstrahlung and that of p-p interactions through $\pi^{0}$-decay for the adopted parameter values are adapted from [62] and [63], respectively. Only the results from the B array configuration are displayed, since they are very similar to those obtained with the E array.

and to get a meaningful spectra with such CTA observations. In Figure 13 (left), we show the simulated energy spectra with different cutoffs as they would be measured by CTA. Simulations for 10 hours of observation time are displayed. To detect Eta Carinae, CTA would need 2-10 hours of observations, depending on the energy cutoff in the spectrum; together with Fermi/LAT data, it should be possible to determine the cutoff energy using a combined fit. However, it would take a longer time to determine the cutoff energy using CTA data alone. The minimum observation time needed to significantly determine the cutoff energy, i.e. to distinguish between a simple power law and a cutoff power law, is established using the likelihood ratio test for the two hypothesis. In Figure 13 (right), we show the resulting significance that a cutoff power law is a better fit to the data than a pure power law versus integration time for the different energies of the cutoff. For this study we simulated 100 spectra for each cutoff energy and for different observation times as shown in the plot. Taking $3 \sigma$ as a limit to distinguish between the two different spectral hypothesis, one can see that 20 hours are enough to detect the cutoff only if it is above $150 \mathrm{GeV}$. For a cutoff $\leq 150 \mathrm{GeV}, 30$ to 50 hours are needed. From our simulations, we can conclude that CTA observation times of $>15$ hours are necessary to make meaningful physics interpretation and modeling, whereas $>20$ hours are necessary to precisely measure the energy cutoff in the spectrum. A proper characterization of the highest energy cutoff will give important clues on the acceleration efficiency of the source, which may be operating close to the limit predicted by diffusive shock acceleration, and on the nature of the radiation mechanism, either leptonic (IC) or hadronic (proton-proton interactions). It is noteworthy that other colliding wind binary systems hosting powerful WR and $\mathrm{O}$ stars may be also powerful non-thermal emitters, as hinted by hard X-ray observations or WR 140 with Suzaku [100].

\section{Summary and conclusion}

The sensitivity of CTA will lead to a very good sampling of light curves and spectra on very short timescales. It will allow as well long source monitoring using subarrays, still with a sensitivity 2-3 times better than any previous instrument operating at VHE energies. In particular, it is noteworthy that CTA will reduce by a factor of a few the errors in the determination of fluxes and spectral indexes. The high sensitivity and good angular resolution will allow also for imaging of possible extended emission in gamma-ray binaries, expected at the termination of the generated outflows. The low energy threshold will also permit to study the maximum particle energy achievable in massive star binaries, trace the effects of electromagnetic cascades in the spectra of gamma-ray binaries, or catch the most luminous part of the spectrum in some sources. Finally, under CTA the population of gamma-ray binaries (and their different subclasses) may easily grow by one order of magnitude, which will imply a strong improvement when looking for patterns and trends, tracing the physical mechanisms behind the non-thermal activity in these sources. For all this, CTA, either in highly sensitive observations of the whole array, or under the more suitable for monitoring subarray mode, will be a tool to obtain the required phenomenological information for deep and accurate modeling of gamma-ray binaries. This can mean a qualitative jump in our physical knowledge of high-energy phenomena in the Galaxy.

Acknowledgments: J.M.P., V.B-R, P.M.-A., J.M., M.R. and V.Z. acknowledge support by DGI of the Spanish Ministerio de Economía y Competitividad (MINECO) under grants AYA2010-21782-C03-01 and FPA2010-22056-C06-02. J.M.P. acknowledges financial support from ICREA Academia. J.M. acknowledges support by MINECO under grant BES-2008- 

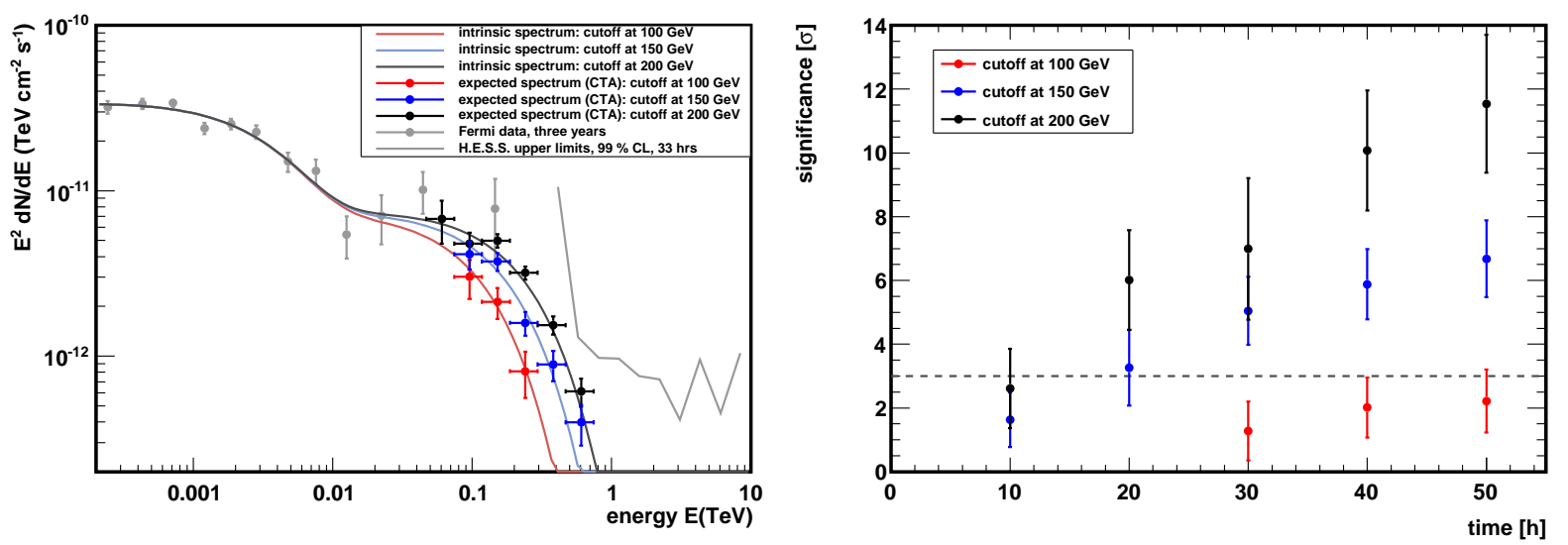

Figure 13: CTA simulations of Eta Carinae. Left: Intrinsic and CTA simulated spectra after 10 hours of observation time with high energy cutoffs at 100, 150 and $200 \mathrm{GeV}$. Right: Significance at which the corresponding cutoffs (color coded, see legend) can be resolved (i. e. are a better fit when compared to a simple power law) versus observation time. The dashed line at $3 \sigma$ is to guide the eye.

004564. M.R. acknowledges financial support from MINECO and European Social Funds through a Ramón y Cajal fellowship. V.Z. was supported by the Spanish MEC through FPU grant AP2006-00077. P.B. has been supported by grant DLR 50 OG 0601 during this work. V.B.-R. acknowledges the support of the European Community under a Marie Curie Intra-European fellowship and financial support from MINECO through a Ramón y Cajal fellowship. D.H., G.P and D.F.T. acknowledge support from the Ministry of Science and the Generalitat de Catalunya, through the grants AYA200907391 and SGR2009-811, as well as by ASPERA-EU through grant EUI-2009-04072. GD acknowledges support from the European Community via contract ERCStG-200911. The research leading to these results has received funding from the European Union's Seventh Framework Programme ([FP7/2007-2013] [FP7/20072011]) under grant agreement n 262053.

\section{References}

[1] F. Aharonian, A. G. Akhperjanian, K.-M. Aye, A. R. BazerBachi, M. Beilicke, W. Benbow, D. Berge, P. Berghaus, K. Bernlöhr, C. Boisson, O. Bolz, I. Braun, F. Breitling, A. M. Brown, J. Bussons Gordo, P. M. Chadwick, L.-M. Chounet, R. Cornils, L. Costamante, B. Degrange, A. Djannati-Ataï, L. O'C. Drury, G. Dubus, D. Emmanoulopoulos, P. Espigat, F. Feinstein, P. Fleury, G. Fontaine, Y. Fuchs, S. Funk, Y. A. Gallant, B. Giebels, S. Gillessen, J. F. Glicenstein, P. Goret, C. Hadjichristidis, M. Hauser, G. Heinzelmann, G. Henri, G. Hermann, J. A. Hinton, W. Hofmann, M. Holleran, D. Horns, O. C. de Jager, S. Johnston, B. Khélifi, J. G. Kirk, N. Komin, A. Konopelko, I. J. Latham, R. Le Gallou, A. Lemière, M. Lemoine-Goumard, N. Leroy, O. MartineauHuynh, T. Lohse, A. Marcowith, C. Masterson, T. J. L.
McComb, M. de Naurois, S. J. Nolan, A. Noutsos, K. J. Orford, J. L. Osborne, M. Ouchrif, M. Panter, G. Pelletier, S. Pita, G. Pühlhofer, M. Punch, B. C. Raubenheimer, M. Raue, J. Raux, S. M. Rayner, I. Redondo, A. Reimer, O. Reimer, J. Ripken, L. Rob, L. Rolland, G. Rowell, V. Sahakian, L. Saugé, S. Schlenker, R. Schlickeiser, C. Schuster, U. Schwanke, M. Siewert, O. Skjæraasen, H. Sol, R. Steenkamp, C. Stegmann, J.-P. Tavernet, R. Terrier, C. G. Théoret, M. Tluczykont, G. Vasileiadis, C. Venter, P. Vincent, H. J. Völk, S. J. Wagner, Discovery of the binary pulsar PSR B1259-63 in very-high-energy gamma rays around periastron with HESS, A\&A 442 (2005) 1-10.

[2] F. Aharonian, A. G. Akhperjanian, G. Anton, U. Barres de Almeida, A. R. Bazer-Bachi, Y. Becherini, et al, Very high energy $\gamma$-ray observations of the binary PSR B1259-63/SS2883 around the 2007 Periastron, A\&A 507 (2009) 389-396.

[3] F. Aharonian, A. G. Akhperjanian, K.-M. Aye, A. R. BazerBachi, M. Beilicke, W. Benbow, D. Berge, P. Berghaus, K. Bernlöhr, C. Boisson, O. Bolz, V. Borrel, I. Braun, F. Breitling, A. M. Brown, J. B. Gordo, P. M. Chadwick, L.-M. Chounet, R. Cornils, L. Costamante, B. Degrange, H. J. Dickinson, A. Djannati-Ataï, L. O. Drury, G. Dubus, D. Emmanoulopoulos, P. Espigat, F. Feinstein, P. Fleury, G. Fontaine, Y. Fuchs, S. Funk, Y. A. Gallant, B. Giebels, S. Gillessen, J. F. Glicenstein, P. Goret, C. Hadjichristidis, M. Hauser, G. Heinzelmann, G. Henri, G. Hermann, J. A. Hinton, W. Hofmann, M. Holleran, D. Horns, A. Jacholkowska, O. C. de Jager, B. Khélifi, N. Komin, A. Konopelko, I. J. Latham, R. Le Gallou, A. Lemière, M. Lemoine-Goumard, N. Leroy, T. Lohse, A. Marcowith, J.-M. Martin, O. Martineau-Huynh, C. Masterson, T. J. L. McComb, M. de Naurois, S. J. Nolan, A. Noutsos, K. J. Orford, J. L. Osborne, M. Ouchrif, M. Panter, G. Pelletier, S. Pita, G. Pühlhofer, M. Punch, B. C. Raubenheimer, M. Raue, J. Raux, S. M. Rayner, A. Reimer, O. Reimer, J. Ripken, L. Rob, L. Rolland, G. Rowell, V. Sahakian, L. Saugé, S. Schlenker, R. Schlickeiser, C. Schuster, U. Schwanke, M. Siewert, H. Sol, D. Spangler, R. Steenkamp, C. Stegmann, J.-P. Tavernet, R. Terrier, C. G. Théoret, M. Tluczykont, G. Vasileiadis, C. Venter, P. Vincent, H. J. Völk, S. J. Wagner, Discovery of Very High Energy Gamma 
Rays Associated with an X-ray Binary, Science 309 (2005) 746-749.

[4] J. Albert, E. Aliu, H. Anderhub, P. Antoranz, A. Armada, M. Asensio, C. Baixeras, J. A. Barrio, M. Bartelt, H. Bartko, D. Bastieri, S. R. Bavikadi, W. Bednarek, K. Berger, C. Bigongiari, A. Biland, E. Bisesi, R. K. Bock, P. Bordas, V. Bosch-Ramon, T. Bretz, I. Britvitch, M. Camara, E. Carmona, A. Chilingarian, S. Ciprini, J. A. Coarasa, S. Commichau, J. L. Contreras, J. Cortina, V. Curtef, V. Danielyan, F. Dazzi, A. De Angelis, R. de los Reyes, B. De Lotto, E. Domingo-Santamaría, D. Dorner, M. Doro, M. Errando, M. Fagiolini, D. Ferenc, E. Fernández, R. Firpo, J. Flix, M. V. Fonseca, L. Font, M. Fuchs, N. Galante, M. Garczarczyk, M. Gaug, M. Giller, F. Goebel, D. Hakobyan, M. Hayashida, T. Hengstebeck, D. Höhne, J. Hose, C. C. Hsu, P. G. Isar, P. Jacon, O. Kalekin, R. Kosyra, D. Kranich, M. Laatiaoui, A. Laille, T. Lenisa, P. Liebing, E. Lindfors, S. Lombardi, F. Longo, J. López, M. López, E. Lorenz, F. Lucarelli, P. Majumdar, G. Maneva, K. Mannheim, O. Mansutti, M. Mariotti, M. Martínez, K. Mase, D. Mazin, C. Merck, M. Meucci, M. Meyer, J. M. Miranda, R. Mirzoyan, S. Mizobuchi, A. Moralejo, K. Nilsson, E. Oña-Wilhelmi, R. Orduña, N. Otte, I. Oya, D. Paneque, R. Paoletti, J. M. Paredes, M. Pasanen, D. Pascoli, F. Pauss, N. Pavel, R. Pegna, M. Persic, L. Peruzzo, A. Piccioli, M. Poller, G. Pooley, E. Prandini, A. Raymers, W. Rhode, M. Ribó, J. Rico, B. Riegel, M. Rissi, A. Robert, G. E. Romero, S. Rügamer, A. Saggion, A. Sánchez, P. Sartori, V. Scalzotto, V. Scapin, R. Schmitt, T. Schweizer, M. Shayduk, K. Shinozaki, S. N. Shore, N. Sidro, A. Sillanpää, D. Sobczynska, A. Stamerra, L. S. Stark, L. Takalo, P. Temnikov, D. Tescaro, M. Teshima, N. Tonello, A. Torres, D. F. Torres, N. Turini, H. Vankov, V. Vitale, R. M. Wagner, T. Wibig, W. Wittek, R. Zanin, J. Zapatero, Variable VeryHigh-Energy Gamma-Ray Emission from the Microquasar LS I +61 303, Science 312 (2006) 1771-1773.

[5] V. A. Acciari, M. Beilicke, G. Blaylock, S. M. Bradbury, J. H. Buckley, V. Bugaev, Y. Butt, K. L. Byrum, O. Celik, A. Cesarini, L. Ciupik, Y. C. K. Chow, P. Cogan, P. Colin, W. Cui, M. K. Daniel, C. Duke, T. Ergin, A. D. Falcone, S. J. Fegan, J. P. Finley, P. Fortin, L. F. Fortson, D. Gall, K. Gibbs, G. H. Gillanders, J. Grube, R. Guenette, D. Hanna, E. Hays, J. Holder, D. Horan, S. B. Hughes, C. M. Hui, T. B. Humensky, P. Kaaret, D. B. Kieda, J. Kildea, A. Konopelko, H. Krawczynski, F. Krennrich, M. J. Lang, S. LeBohec, K. Lee, G. Maier, A. McCann, M. McCutcheon, J. Millis, P. Moriarty, R. Mukherjee, T. Nagai, R. A. Ong, D. Pandel, J. S. Perkins, F. Pizlo, M. Pohl, J. Quinn, K. Ragan, P. T. Reynolds, H. J. Rose, M. Schroedter, G. H. Sembroski, A. W. Smith, D. Steele, S. P. Swordy, J. A. Toner, L. Valcarcel, V. V. Vassiliev, R. Wagner, S. P. Wakely, J. E. Ward, T. C. Weekes, A. Weinstein, R. J. White, D. A. Williams, S. A. Wissel, M. Wood, B. Zitzer, VERITAS Observations of the $\gamma$-Ray Binary LS I +61303 , ApJ 679 (2008) 1427-1432.

[6] F. A. Aharonian, A. G. Akhperjanian, A. R. Bazer-Bachi, B. Behera, M. Beilicke, W. Benbow, D. Berge, K. Bernlöhr, C. Boisson, O. Bolz, V. Borrel, I. Braun, E. Brion, A. M. Brown, R. Bühler, I. Büsching, T. Boutelier, S. Carrigan, P. M. Chadwick, L.-M. Chounet, G. Coignet, R. Cornils, L. Costamante, B. Degrange, H. J. Dickinson, A. Djannati-Ataï, W. Domainko, L. O'C. Drury, G. Dubus, K. Egberts, D. Emmanoulopoulos, P. Espigat, C. Farnier, F. Feinstein, A. Fiasson, A. Förster, G. Fontaine, S. Funk, S. Funk, M. Füßling, Y. A. Gallant, B. Giebels, J. F. Glicenstein, B. Glück, P. Goret, C. Hadjichristidis, D. Hauser, M. Hauser, G. Heinzelmann, G. Henri, G. Hermann, J. A. Hinton, A. Hoffmann, W. Hof- mann, M. Holleran, S. Hoppe, D. Horns, A. Jacholkowska, O. C. de Jager, E. Kendziorra, M. Kerschhaggl, B. Khélifi, N. Komin, K. Kosack, G. Lamanna, I. J. Latham, R. Le Gallou, A. Lemière, M. Lemoine-Goumard, T. Lohse, J. M. Martin, O. Martineau-Huynh, A. Marcowith, C. Masterson, G. Maurin, T. J. L. McComb, E. Moulin, M. de Naurois, D. Nedbal, S. J. Nolan, A. Noutsos, J.-P. Olive, K. J. Orford, J. L. Osborne, M. Panter, G. Pedaletti, G. Pelletier, P.O. Petrucci, S. Pita, G. Pühlhofer, M. Punch, S. Ranchon, B. C. Raubenheimer, M. Raue, S. M. Rayner, O. Reimer, J. Ripken, L. Rob, L. Rolland, S. Rosier-Lees, G. Rowell, J. Ruppel, V. Sahakian, A. Santangelo, L. Saugé, S. Schlenker, R. Schlickeiser, R. Schröder, U. Schwanke, S. Schwarzburg, S. Schwemmer, A. Shalchi, H. Sol, D. Spangler, R. Steenkamp, C. Stegmann, G. Superina, P. H. Tam, J.-P. Tavernet, R. Terrier, M. Tluczykont, C. van Eldik, G. Vasileiadis, C. Venter, J. P. Vialle, P. Vincent, H. J. Völk, S. J. Wagner, M. Ward, Y. Moriguchi, Y. Fukui, Discovery of a point-like very-highenergy $\gamma$-ray source in Monoceros, A\&A 469 (2007) L1-L4.

[7] J. Albert, E. Aliu, H. Anderhub, P. Antoranz, A. Armada, C. Baixeras, J. A. Barrio, H. Bartko, D. Bastieri, J. K. Becker, W. Bednarek, K. Berger, C. Bigongiari, A. Biland, R. K. Bock, P. Bordas, V. Bosch-Ramon, T. Bretz, I. Britvitch, M. Camara, E. Carmona, A. Chilingarian, J. A. Coarasa, S. Commichau, J. L. Contreras, J. Cortina, M. T. Costado, V. Curtef, V. Danielyan, F. Dazzi, A. De Angelis, C. Delgado, R. de los Reyes, B. De Lotto, E. Domingo-Santamaría, D. Dorner, M. Doro, M. Errando, M. Fagiolini, D. Ferenc, E. Fernández, R. Firpo, J. Flix, M. V. Fonseca, L. Font, M. Fuchs, N. Galante, R. J. García-López, M. Garczarczyk, M. Gaug, M. Giller, F. Goebel, D. Hakobyan, M. Hayashida, T. Hengstebeck, A. Herrero, D. Höhne, J. Hose, C. C. Hsu, P. Jacon, T. Jogler, R. Kosyra, D. Kranich, R. Kritzer, A. Laille, E. Lindfors, S. Lombardi, F. Longo, J. López, M. López, E. Lorenz, P. Majumdar, G. Maneva, K. Mannheim, O. Mansutti, M. Mariotti, M. Martínez, D. Mazin, C. Merck, M. Meucci, M. Meyer, J. M. Miranda, R. Mirzoyan, S. Mizobuchi, A. Moralejo, D. Nieto, K. Nilsson, J. Ninkovic, E. Oña-Wilhelmi, N. Otte, I. Oya, M. Panniello, R. Paoletti, J. M. Paredes, M. Pasanen, D. Pascoli, F. Pauss, R. Pegna, M. Persic, L. Peruzzo, A. Piccioli, E. Prandini, N. Puchades, A. Raymers, W. Rhode, M. Ribó, J. Rico, M. Rissi, A. Robert, S. Rügamer, A. Saggion, T. Saito, A. Sánchez, P. Sartori, V. Scalzotto, V. Scapin, R. Schmitt, T. Schweizer, M. Shayduk, K. Shinozaki, S. N. Shore, N. Sidro, A. Sillanpää, D. Sobczynska, A. Stamerra, L. S. Stark, L. Takalo, P. Temnikov, D. Tescaro, M. Teshima, D. F. Torres, N. Turini, H. Vankov, V. Vitale, R. M. Wagner, T. Wibig, W. Wittek, F. Zandanel, R. Zanin, J. Zapatero, Very High Energy Gamma-Ray Radiation from the Stellar Mass Black Hole Binary Cygnus X-1, ApJ 665 (2007) L51-L54.

[8] J. M. Paredes, Gamma-ray binaries: microquasars and binary systems with pulsar, ArXiv e-prints:1101.4843 (2011).

[9] Fermi LAT Collaboration, M. Ackermann, M. Ajello, J. Ballet, G. Barbiellini, D. Bastieri, et al, Periodic Emission from the Gamma-Ray Binary 1FGL J1018.6-5856, Science 335 (2012) $189-$

[10] E. de Ona Wilhelmi, R. Terrier, R. Chaves, O. de Jager, A. Djannati-Ataï, A. Abramowski, F. Acero, F. Aharonian, A. G. Akhperjanian, G. Anton, et al, Observations of the supernova remnant SNR G284.3-1.8 from X-rays to VHE gamma-rays with XMM-Newton, Fermi and H.E.S.S., in: 38th COSPAR Scientific Assembly, volume 38, pp. 2803-+.

[11] V. Bosch-Ramon, Spectral energy distribution of gamma-ray binaries: sources and processes, Mem. Soc. Astron. Italiana 82 (2011) 182-+. 
[12] G. Dubus, J. L. Contreras, S. Funk, Y. Gallant, T. Hassan, J. Hinton, Y. Inoue, J. Knödlseder, P. Martin, N. Mirabal, M. de Naurois, M. Renaud, f. t. CTA consortium, Surveys with the Cherenkov Telescope Array, ArXiv e-prints (2012).

[13] D. Khangulyan, F. Aharonian, V. Bosch-Ramon, On the formation of TeV radiation in LS 5039, MNRAS 383 (2008) 467478.

[14] M. Actis, G. Agnetta, F. Aharonian, A. Akhperjanian, J. Aleksić, E. Aliu, D. Allan, I. Allekotte, F. Antico, L. A. Antonelli, et al., Design concepts for the Cherenkov Telescope Array CTA: an advanced facility for ground-based high-energy gamma-ray astronomy, Experimental Astronomy 32 (2011) 193-316.

[15] M. Tavani, F. Lucarelli, C. Pittori, F. Verrecchia, A. Bulgarelli, F. Gianotti, M. Trifoglio, E. Striani, S. Sabatini, G. Piano, A. Argan, A. Trois, G. de Paris, V. Vittorini, E. Costa, I. Donnarumma, M. Feroci, L. Pacciani, E. Del Monte, F. Lazzarotto, P. Soffitta, Y. Evangelista, I. Lapshov, A. Chen, A. Giuliani, M. Marisaldi, G. Di Cocco, C. Labanti, F. Fuschino, M. Galli, P. Caraveo, S. Mereghetti, F. Perotti, G. Pucella, M. Rapisarda, A. Pellizzoni, M. Pilia, G. Barbiellini, F. Longo, P. Picozza, A. Morselli, M. Prest, P. Lipari, D. Zanello, S. Vercellone, F. D'Ammando, P. W. Cattaneo, A. Rappoldi, P. Giommi, P. Santolamazza, S. Colafrancesco, L. Salotti, AGILE detection of transient gamma-ray emission from the PSR B1259-63 region, The Astronomer's Telegram 2772 (2010) 1.

[16] P. H. T. Tam, R. H. H. Huang, J. Takata, C. Y. Hui, A. K. H. Kong, K. S. Cheng, Discovery of GeV $\gamma$-ray Emission from PSR B1259-63/LS 2883, ApJ 736 (2011) L10.

[17] A. A. Abdo, M. Ackermann, M. Ajello, A. Allafort, J. Ballet, et al., Discovery of High-energy Gamma-ray Emission from the Binary System PSR B1259-63/LS 2883 around Periastron with Fermi, ApJ 736 (2011) L11.

[18] J. Moldón, S. Johnston, M. Ribó, J. M. Paredes, A. T. Deller, Discovery of Extended and Variable Radio Structure from the Gamma-ray Binary System PSR B1259-63/LS 2883, ApJ 732 (2011) L10+.

[19] M. Tavani, J. Arons, Theory of High-Energy Emission from the Pulsar/Be Star System PSR 1259-63. I. Radiation Mechanisms and Interaction Geometry, ApJ 477 (1997) 439-+.

[20] G. Dubus, Gamma-ray binaries: pulsars in disguise?, A\&A 456 (2006) 801-817.

[21] D. Khangulyan, S. Hnatic, F. Aharonian, S. Bogovalov, TeV light curve of PSR B1259-63/SS2883, MNRAS 380 (2007) 320-330.

[22] F. Aharonian, A. G. Akhperjanian, A. R. Bazer-Bachi, M. Beilicke, W. Benbow, D. Berge, K. Bernlöhr, C. Boisson, O. Bolz, V. Borrel, I. Braun, A. M. Brown, R. Bühler, I. Büsching, S. Carrigan, P. M. Chadwick, L.-M. Chounet, R. Cornils, L. Costamante, B. Degrange, H. J. Dickinson, A. Djannati-Ataï, L. O'C. Drury, G. Dubus, K. Egberts, D. Emmanoulopoulos, P. Espigat, F. Feinstein, E. Ferrero, A. Fiasson, G. Fontaine, S. Funk, S. Funk, M. Füßling, Y. A. Gallant, B. Giebels, J. F. Glicenstein, P. Goret, C. Hadjichristidis, D. Hauser, M. Hauser, G. Heinzelmann, G. Henri, G. Hermann, J. A. Hinton, A. Hoffmann, W. Hofmann, M. Holleran, D. Horns, A. Jacholkowska, O. C. de Jager, E. Kendziorra, B. Khélifi, N. Komin, A. Konopelko, K. Kosack, I. J. Latham, R. Le Gallou, A. Lemière, M. Lemoine-Goumard, T. Lohse, J. M. Martin, O. Martineau-Huynh, A. Marcowith, C. Masterson, G. Maurin, T. J. L. McComb, E. Moulin, M. de Naurois, D. Nedbal, S. J. Nolan, A. Noutsos, K. J. Orford, J. L. Osborne, M. Ouchrif, M. Panter, G. Pelletier, S. Pita, G. Pühlhofer, M. Punch, B. C. Raubenheimer, M. Raue, S. M. Rayner, A. Reimer, O. Reimer, J. Ripken, L. Rob,
L. Rolland, G. Rowell, V. Sahakian, A. Santangelo, L. Saugé, S. Schlenker, R. Schlickeiser, R. Schröder, U. Schwanke, S. Schwarzburg, A. Shalchi, H. Sol, D. Spangler, F. Spanier, R. Steenkamp, C. Stegmann, G. Superina, J.-P. Tavernet, R. Terrier, M. Tluczykont, C. van Eldik, G. Vasileiadis, C. Venter, P. Vincent, H. J. Völk, S. J. Wagner, M. Ward, 3.9 day orbital modulation in the TeV $\gamma$-ray flux and spectrum from the X-ray binary LS 5039, A\&A 460 (2006) 743-749.

[23] J. Albert, E. Aliu, H. Anderhub, L. A. Antonelli, P. Antoranz, M. Backes, C. Baixeras, J. A. Barrio, H. Bartko, D. Bastieri, J. K. Becker, W. Bednarek, K. Berger, E. Bernardini, C. Bigongiari, A. Biland, R. K. Bock, G. Bonnoli, P. Bordas, V. Bosch-Ramon, T. Bretz, I. Britvitch, M. Camara, E. Carmona, A. Chilingarian, S. Commichau, J. L. Contreras, J. Cortina, M. T. Costado, S. Covino, V. Curtef, F. Dazzi, A. DeAngelis, E. DeCea del Pozo, R. de los Reyes, B. DeLotto, M. DeMaria, F. DeSabata, C. Delgado Mendez, A. Dominguez, D. Dorner, M. Doro, M. Errando, M. Fagiolini, D. Ferenc, E. Fernández, R. Firpo, M. V. Fonseca, L. Font, N. Galante, R. J. García López, M. Garczarczyk, M. Gaug, F. Goebel, M. Hayashida, A. Herrero, D. Höhne, J. Hose, C. C. Hsu, S. Huber, T. Jogler, D. Kranich, A. La Barbera, A. Laille, E. Leonardo, E. Lindfors, S. Lombardi, F. Longo, M. López, E. Lorenz, P. Majumdar, G. Maneva, N. Mankuzhiyil, K. Mannheim, L. Maraschi, M. Mariotti, M. Martínez, D. Mazin, M. Meucci, M. Meyer, J. M. Miranda, R. Mirzoyan, S. Mizobuchi, M. Moles, A. Moralejo, D. Nieto, K. Nilsson, J. Ninkovic, N. Otte, I. Oya, M. Panniello, R. Paoletti, J. M. Paredes, M. Pasanen, D. Pascoli, F. Pauss, R. G. Pegna, M. A. Perez-Torres, M. Persic, L. Peruzzo, A. Piccioli, F. Prada, E. Prandini, N. Puchades, A. Raymers, W. Rhode, M. Ribó, J. Rico, M. Rissi, A. Robert, S. Rügamer, A. Saggion, T. Y. Saito, M. Salvati, M. Sanchez-Conde, P. Sartori, K. Satalecka, V. Scalzotto, V. Scapin, R. Schmitt, T. Schweizer, M. Shayduk, K. Shinozaki, S. N. Shore, N. Sidro, A. Sierpowska-Bartosik, A. Sillanpää, D. Sobczynska, F. Spanier, A. Stamerra, L. S. Stark, L. Takalo, F. Tavecchio, P. Temnikov, D. Tescaro, M. Teshima, M. Tluczykont, D. F. Torres, N. Turini, H. Vankov, A. Venturini, V. Vitale, R. M. Wagner, W. Wittek, V. Zabalza, F. Zandanel, R. Zanin, J. Zapatero, Periodic Very High Energy $\gamma$-Ray Emission from LS I +61303 Observed with the MAGIC Telescope, ApJ 693 (2009) 303-310.

[24] A. A. Abdo, M. Ackermann, M. Ajello, W. B. Atwood, M. Axelsson, et al., Fermi/LAT observations of LS 5039, ApJ 706 (2009) L56-L61.

[25] A. A. Abdo, M. Ackermann, M. Ajello, W. B. Atwood, M. Axelsson, et al., Fermi LAT Observations of LS I +61303: First Detection of an Orbital Modulation in GeV Gamma Rays, ApJ 701 (2009) L123-L128.

[26] J. Casares, M. Ribó, I. Ribas, J. M. Paredes, J. Martí, A. Herrero, A possible black hole in the $\gamma$-ray microquasar LS 5039, MNRAS 364 (2005) 899-908.

[27] J. Casares, I. Ribas, J. M. Paredes, J. Martí, C. Allende Prieto, Orbital parameters of the microquasar LS I +61 303, MNRAS 360 (2005) 1105-1109.

[28] J. M. Paredes, J. Martí, M. Ribó, M. Massi, Discovery of a High-Energy Gamma-Ray-Emitting Persistent Microquasar, Science 288 (2000) 2340-2342.

[29] M. Ribó, J. M. Paredes, J. Moldón, J. Martí, M. Massi, The changing milliarcsecond radio morphology of the gamma-ray binary LS 5039, A\&A 481 (2008) 17-20.

[30] V. Dhawan, A. Mioduszewski, M. Rupen, LS I +61 303 is a Be-Pulsar binary, not a Microquasar, in: VI Microquasar Workshop: Microquasars and Beyond. 
[31] J. Moldón, M. Ribó, J. M. Paredes, Periodic morphological changes in the radio structure of the gamma-ray binary LS 5039, A\&A, in press, ArXiv e-prints:1209.6073 (2012).

[32] D. Hadasch, D. F. Torres, T. Tanaka, R. H. D. Corbet, A. B. Hill, R. Dubois, G. Dubus, T. Glanzman, S. Corbel, J. Li, Y. P. Chen, S. Zhang, G. A. Caliandro, M. Kerr, J. L. Richards, W. Max-Moerbeck, A. Readhead, G. Pooley, Long-term Monitoring of the High-energy $\gamma$-Ray Emission from LS I +61303 and LS 5039, ApJ 749 (2012) 54.

[33] T. Takahashi, T. Kishishita, Y. Uchiyama, T. Tanaka, K. Yamaoka, D. Khangulyan, F. A. Aharonian, V. Bosch-Ramon, J. A. Hinton, Study of the Spectral and Temporal Characteristics of X-Ray Emission of the Gamma-Ray Binary LS 5039 with Suzaku, ApJ 697 (2009) 592-600.

[34] W. Bednarek, Cascade initiated by VHE $\gamma$-rays in the radiation field of a close massive companion., A\&A 322 (1997) 523532 .

[35] W. Bednarek, Escape of VHE gamma-rays from close massive binary Centauri X-3, A\&A 362 (2000) 646-654.

[36] W. Bednarek, Propagation of very high energy $\gamma$-rays inside massive binaries LS 5039 and LSI +61 303, MNRAS 368 (2006) 579-591.

[37] J. A. Hinton, J. L. Skilton, S. Funk, J. Brucker, F. A. Aharonian, G. Dubus, A. Fiasson, Y. Gallant, W. Hofmann, A. Marcowith, O. Reimer, HESS J0632+057: A New Gamma-Ray Binary?, ApJ 690 (2009) L101-L104.

[38] V. A. Acciari, E. Aliu, T. Arlen, M. Beilicke, W. Benbow, D. Boltuch, S. M. Bradbury, J. H. Buckley, V. Bugaev, K. Byrum, A. Cannon, A. Cesarini, A. Cesarini, Y. C. Chow, L. Ciupik, P. Cogan, R. Dickherber, C. Duke, T. Ergin, A. Falcone, S. J. Fegan, J. P. Finley, G. Finnegan, P. Fortin, L. Fortson, A. Furniss, K. Gibbs, G. H. Gillanders, J. Grube, R. Guenette, G. Gyuk, D. Hanna, J. Holder, D. Horan, C. M. Hui, T. B. Humensky, A. Imran, P. Kaaret, N. Karlsson, M. Kertzman, D. Kieda, J. Kildea, A. Konopelko, H. Krawczynski, F. Krennrich, M. J. Lang, S. LeBohec, S. LeBohec, G. Maier, A. McCann, M. McCutcheon, J. Millis, J. Millis, P. Moriarty, R. Mukherjee, R. A. Ong, A. N. Otte, D. Pandel, J. S. Perkins, D. Petry, M. Pohl, J. Quinn, K. Ragan, L. C. Reyes, P. T. Reynolds, H. J. Rose, M. Schroedter, G. H. Sembroski, A. W. Smith, D. Steele, S. Swordy, M. Theiling, J. A. Toner, A. Varlotta, V. V. Vassiliev, S. Vincent, R. G. Wagner, S. P. Wakely, J. E. Ward, T. C. Weekes, A. Weinstein, T. Weisgarber, D. A. Williams, S. Wissel, M. Wood, Evidence for Long-Term Gamma-Ray and X-Ray Variability from the Unidentified TeV Source HESS J0632+057, ApJ 698 (2009) L94-L97.

[39] R. A. Ong, VERITAS Reports Increased Activity of HESS J0632+057 in Very High Energy Gamma Rays, The Astronomer's Telegram 3153 (2011) 1-+.

[40] The MAGIC Collaboration, J. Aleksić, E. A. Alvarez, L. A. Antonelli, P. Antoranz, et al, Detection of VHE \gamma-rays from HESS J0632+057 during the 2011 February X-ray outburst with the MAGIC Telescopes, ArXiv e-prints (2012).

[41] S. D. Bongiorno, A. D. Falcone, M. Stroh, J. Holder, J. L. Skilton, J. A. Hinton, N. Gehrels, J. Grube, A New TeV Binary: The Discovery of an Orbital Period in HESS J0632+057, ApJ 737 (2011) L11+.

[42] H. Anderhub, L. A. Antonelli, P. Antoranz, M. Backes, C. Baixeras, S. Balestra, J. A. Barrio, D. Bastieri, J. Becerra González, J. K. Becker, et al, Correlated X-Ray and Very High Energy Emission in the Gamma-Ray Binary LS I +61 303, ApJ 706 (2009) L27-L32.

[43] J. Casares, M. Ribó, I. Ribas, J. M. Paredes, F. Vilardell, I. Negueruela, On the binary nature of the $\gamma$-ray sources AGL
J2241+4454 (= MWC 656) and HESS J0632+057 (= MWC 148), MNRAS 421 (2012) 1103-1112.

[44] N. Rea, D. F. Torres, The TeV Binary HESS J0632+057 in the Low and High X-Ray State, ApJ 737 (2011) L12+.

[45] J. Moldón, M. Ribó, J. M. Paredes, Revealing the extended radio emission from the gamma-ray binary HESS J0632+057, A\&A 533 (2011) L7+.

[46] M. Tavani, A. Bulgarelli, G. Piano, S. Sabatini, E. Striani, Y. Evangelista, A. Trois, G. Pooley, S. Trushkin, N. A. Nizhelskij, M. McCollough, K. I. I. Koljonen, G. Pucella, A. Giuliani, A. W. Chen, E. Costa, V. Vittorini, M. Trifoglio, F. Gianotti, A. Argan, G. Barbiellini, P. Caraveo, P. W. Cattaneo, V. Cocco, T. Contessi, F. D’Ammando, E. Del Monte, G. de Paris, G. Di Cocco, G. di Persio, I. Donnarumma, M. Feroci, A. Ferrari, F. Fuschino, M. Galli, C. Labanti, I. Lapshov, F. Lazzarotto, P. Lipari, F. Longo, E. Mattaini, M. Marisaldi, M. Mastropietro, A. Mauri, S. Mereghetti, E. Morelli, A. Morselli, L. Pacciani, A. Pellizzoni, F. Perotti, P. Picozza, M. Pilia, M. Prest, M. Rapisarda, A. Rappoldi, E. Rossi, A. Rubini, E. Scalise, P. Soffitta, E. Vallazza, S. Vercellone, A. Zambra, D. Zanello, C. Pittori, F. Verrecchia, P. Giommi, S. Colafrancesco, P. Santolamazza, A. Antonelli, L. Salotti, Extreme particle acceleration in the microquasar CygnusX-3, Nature 462 (2009) 620623.

[47] Fermi LAT Collaboration, A. A. Abdo, M. Ackermann, M. Ajello, M. Axelsson, L. Baldini, et al, Modulated HighEnergy Gamma-Ray Emission from the Microquasar Cygnus X-3, Science 326 (2009) 1512-.

[48] S. Sabatini, M. Tavani, E. Striani, A. Bulgarelli, V. Vittorini, G. Piano, E. Del Monte, M. Feroci, F. de Pasquale, M. Trifoglio, F. Gianotti, A. Argan, G. Barbiellini, P. Caraveo, P. W. Cattaneo, A. W. Chen, F. D'Ammando, E. Costa, G. De Paris, G. Di Cocco, I. Donnarumma, Y. Evangelista, A. Ferrari, M. Fiorini, F. Fuschino, M. Galli, A. Giuliani, M. Giusti, C. Labanti, F. Lazzarotto, P. Lipari, F. Longo, M. Marisaldi, S. Mereghetti, E. Morelli, E. Moretti, A. Morselli, L. Pacciani, A. Pellizzoni, F. Perotti, P. Picozza, M. Pilia, G. Pucella, M. Prest, M. Rapisarda, A. Rappoldi, A. Rubini, E. Scalise, P. Soffitta, A. Trois, E. Vallazza, S. Vercellone, A. Zambra, D. Zanello, C. Pittori, F. Verrecchia, P. Santolamazza, P. Giommi, S. Colafrancesco, L. A. Antonelli, L. Salotti, Episodic Transient Gamma-ray Emission from the Microquasar Cygnus X-1, ApJ 712 (2010) L10-L15.

[49] J. Albert, E. Aliu, H. Anderhub, P. Antoranz, A. Armada, C. Baixeras, J. A. Barrio, H. Bartko, D. Bastieri, J. K. Becker, W. Bednarek, K. Berger, C. Bigongiari, A. Biland, R. K. Bock, P. Bordas, V. Bosch-Ramon, T. Bretz, I. Britvitch, M. Camara, E. Carmona, A. Chilingarian, J. A. Coarasa, S. Commichau, J. L. Contreras, J. Cortina, M. T. Costado, V. Curtef, V. Danielyan, F. Dazzi, A. De Angelis, C. Delgado, R. de los Reyes, B. De Lotto, E. Domingo-Santamaría, D. Dorner, M. Doro, M. Errando, M. Fagiolini, D. Ferenc, E. Fernández, R. Firpo, J. Flix, M. V. Fonseca, L. Font, M. Fuchs, N. Galante, R. J. García-López, M. Garczarczyk, M. Gaug, M. Giller, F. Goebel, D. Hakobyan, M. Hayashida, T. Hengstebeck, A. Herrero, D. Höhne, J. Hose, C. C. Hsu, P. Jacon, T. Jogler, R. Kosyra, D. Kranich, R. Kritzer, A. Laille, E. Lindfors, S. Lombardi, F. Longo, J. López, M. López, E. Lorenz, P. Majumdar, G. Maneva, K. Mannheim, O. Mansutti, M. Mariotti, M. Martínez, D. Mazin, C. Merck, M. Meucci, M. Meyer, J. M. Miranda, R. Mirzoyan, S. Mizobuchi, A. Moralejo, D. Nieto, K. Nilsson, J. Ninkovic, E. Oña-Wilhelmi, N. Otte, I. Oya, M. Panniello, R. Paoletti, J. M. Paredes, M. Pasanen, D. Pascoli, F. Pauss, R. Pegna, M. Persic, L. Peruzzo, A. Piccioli, E. Prandini, N. Puchades, A. Raymers, W. Rhode, 
M. Ribó, J. Rico, M. Rissi, A. Robert, S. Rügamer, A. Saggion, T. Saito, A. Sánchez, P. Sartori, V. Scalzotto, V. Scapin, R. Schmitt, T. Schweizer, M. Shayduk, K. Shinozaki, S. N. Shore, N. Sidro, A. Sillanpää, D. Sobczynska, A. Stamerra, L. S. Stark, L. Takalo, P. Temnikov, D. Tescaro, M. Teshima, D. F. Torres, N. Turini, H. Vankov, V. Vitale, R. M. Wagner, T. Wibig, W. Wittek, F. Zandanel, R. Zanin, J. Zapatero, Very High Energy Gamma-Ray Radiation from the Stellar Mass Black Hole Binary Cygnus X-1, ApJ 665 (2007) L51-L54.

[50] J. Aleksić, L. A. Antonelli, P. Antoranz, M. Backes, C. Baixeras, J. A. Barrio, D. Bastieri, J. Becerra González, W. Bednarek, A. Berdyugin, K. Berger, E. Bernardini, A. Biland, O. Blanch, R. K. Bock, A. Boller, G. Bonnoli, P. Bordas, D. Borla Tridon, V. Bosch-Ramon, D. Bose, I. Braun, T. Bretz, D. Britzger, M. Camara, E. Carmona, A. Carosi, P. Colin, J. L. Contreras, J. Cortina, M. T. Costado, S. Covino, F. Dazzi, A. De Angelis, E. De Cea del Pozo, B. De Lotto, M. De Maria, F. De Sabata, C. Delgado Mendez, M. Doert, A. Domínguez, D. Dominis Prester, D. Dorner, M. Doro, D. Elsaesser, M. Errando, D. Ferenc, M. V. Fonseca, L. Font, R. J. García López, M. Garczarczyk, M. Gaug, N. Godinovic, F. Göebel, D. Hadasch, A. Herrero, D. Hildebrand, D. Höhne-Mönch, J. Hose, D. Hrupec, C. C. Hsu, T. Jogler, S. Klepser, T. Krähenbühl, D. Kranich, A. La Barbera, A. Laille, E. Leonardo, E. Lindfors, S. Lombardi, F. Longo, M. López, E. Lorenz, P. Majumdar, G. Maneva, N. Mankuzhiyil, K. Mannheim, L. Maraschi, M. Mariotti, M. Martínez, D. Mazin, M. Meucci, J. M. Miranda, R. Mirzoyan, H. Miyamoto, J. Moldón, M. Moles, A. Moralejo, D. Nieto, K. Nilsson, J. Ninkovic, R. Orito, I. Oya, S. Paiano, R. Paoletti, J. M. Paredes, S. Partini, M. Pasanen, D. Pascoli, F. Pauss, R. G. Pegna, M. A. Perez-Torres, M. Persic, L. Peruzzo, F. Prada, E. Prandini, N. Puchades, I. Puljak, I. Reichardt, W. Rhode, M. Ribó, J. Rico, M. Rissi, S. Rügamer, A. Saggion, K. Saito, T. Y. Saito, M. Salvati, M. SánchezConde, K. Satalecka, V. Scalzotto, V. Scapin, C. Schultz, T. Schweizer, M. Shayduk, S. N. Shore, A. SierpowskaBartosik, A. Sillanpää, J. Sitarek, D. Sobczynska, F. Spanier, S. Spiro, A. Stamerra, B. Steinke, J. C. Struebig, T. Suric, L. Takalo, F. Tavecchio, P. Temnikov, T. Terzic, D. Tescaro, M. Teshima, D. F. Torres, H. Vankov, R. M. Wagner, Q. Weitzel, V. Zabalza, F. Zandanel, R. Zanin, The MAGIC Collaboration, Magic Constraints on $\gamma$-ray Emission from Cygnus X-3, ApJ 721 (2010) 843-855.

[51] R. Zanin, T. Sayto, V. Zabalza, P. Bordas, T. Jogler, J. Cortina, J. M. Paredes, M. Ribó, Javier Rico for the MAGIC collaboration, Search for VHE signals from microquarsars with MAGIC, ArXiv e-prints:1110.1581 (2011).

[52] Hess Collaboration, F. Acero, F. Aharonian, A. G. Akhperjanian, G. Anton, U. Barres de Almeida, et al, HESS upper limits on very high energy gamma-ray emission from the microquasar GRS 1915+105, A\&A 508 (2009) 1135-1140.

[53] J. Aleksić, E. A. Alvarez, L. A. Antonelli, P. Antoranz, M. Asensio, et al, A Search for Very High Energy GammaRay Emission from Scorpius X-1 with the Magic Telescopes, ApJ 735 (2011) L5+.

[54] G. S. Vila, G. E. Romero, Leptonic/hadronic models for electromagnetic emission in microquasars: the case of GX 339-4, MNRAS 403 (2010) 1457-1468.

[55] M. M. Kaufman Bernadó, G. E. Romero, I. F. Mirabel, Precessing microblazars and unidentified gamma-ray sources, A\&A 385 (2002) L10-L13.

[56] G. E. Romero, D. F. Torres, M. M. Kaufman Bernadó, I. F. Mirabel, Hadronic gamma-ray emission from windy microquasars, A\&A 410 (2003) L1-L4.
[57] I. V. Moskalenko, S. Karakula, W. Tkaczyk, Cygnus X-3 lightcurve model in the TeV energy region, MNRAS 260 (1993) 681-685.

[58] G. Dubus, Gamma-ray absorption in massive X-ray binaries, A\&A 451 (2006) 9-18.

[59] M. Perucho, V. Bosch-Ramon, D. Khangulyan, 3D simulations of wind-jet interaction in massive X-ray binaries, A\&A 512 (2010) L4.

[60] V. Bosch-Ramon, D. Khangulyan, Monte Carlo Simulations of Radio Emitting Secondaries in $\gamma$-Ray Binaries, PASJ 63 (2011) 1023-1033.

[61] S. Heinz, Radio lobe dynamics and the environment of microquasars, A\&A 388 (2002) L40-L43.

[62] P. Bordas, V. Bosch-Ramon, J. M. Paredes, M. Perucho, Nonthermal emission from microquasar/ISM interaction, A\&A 497 (2009) 325-334.

[63] J. F. Zhang, Y. G. Feng, Non-thermal emission from the termination of microquasar jets interacting with the interstellar medium, MNRAS 410 (2011) 978-983.

[64] V. Bosch-Ramon, M. V. Barkov, Large-scale flow dynamics and radiation in pulsar $\gamma$-ray binaries, A\&A 535 (2011) A20.

[65] S. M. Dougherty, A. J. Beasley, M. J. Claussen, B. A. Zauderer, N. J. Bolingbroke, High-Resolution Radio Observations of the Colliding-Wind Binary WR 140, ApJ 623 (2005) 447459.

[66] Y. Sugawara, Y. Maeda, Y. Tsuboi, K. Hamaguchi, Suzaku monitoring of the Wolf-Rayet binary WR140, X-ray Astronomy 2009; Present Status, Multi-Wavelength Approach and Future Perspectives 1248 (2010) 9-12.

[67] D. Eichler, V. Usov, Particle acceleration and nonthermal radio emission in binaries of early-type stars, ApJ 402 (1993) 271279.

[68] P. Benaglia, G. E. Romero, I. R. Stevens, D. F. Torres, Can the gamma-ray source $3 \mathrm{EG} \mathrm{J} 2033+4118$ be produced by the stellar system Cygnus OB2 No. 5?, A\&A 366 (2001) 605-611.

69] A. Damineli, D. J. Hillier, M. F. Corcoran, O. Stahl, R. S. Levenhagen, N. V. Leister, J. H. Groh, M. Teodoro, J. F. Albacete Colombo, F. Gonzalez, J. Arias, H. Levato, M. Grosso, N. Morrell, R. Gamen, G. Wallerstein, V. Niemela, The periodicity of the $\eta$ Carinae events, MNRAS 384 (2008) 1649-1656.

[70] A. Reimer, M. Pohl, O. Reimer, Nonthermal High-Energy Emission from Colliding Winds of Massive Stars, ApJ 644 (2006) 1118-1144.

[71] W. Bednarek, J. Pabich, High-energy radiation from the massive binary system Eta Carinae, A\&A 530 (2011) A49+.

[72] A. A. Abdo, M. Ackermann, M. Ajello, A. Allafort, L. Baldini, J. Ballet, G. Barbiellini, D. Bastieri, K. Bechtol, R. Bellazzini, et al, Fermi Large Area Telescope Observation of a Gammaray Source at the Position of Eta Carinae, ApJ 723 (2010) 649-657.

[73] K. Reitberger, O. Reimer, A. Reimer, M. Werner, K. Egberts, H. Takahashi, for the Fermi LAT collaboration, Gamma-ray follow-up studies on Eta Carinae, ArXiv e-prints (2012).

[74] M. Tavani, S. Sabatini, E. Pian, A. Bulgarelli, P. Caraveo, R. F. Viotti, M. F. Corcoran, A. Giuliani, C. Pittori, F. Verrecchia, S. Vercellone, S. Mereghetti, A. Argan, G. Barbiellini, F. Boffelli, P. W. Cattaneo, A. W. Chen, V. Cocco, F. D'Ammando, E. Costa, G. DeParis, E. Del Monte, G. Di Cocco, I. Donnarumma, Y. Evangelista, A. Ferrari, M. Feroci, M. Fiorini, T. Froysland, F. Fuschino, M. Galli, F. Gianotti, C. Labanti, I. Lapshov, F. Lazzarotto, P. Lipari, F. Longo, M. Marisaldi, M. Mastropietro, E. Morelli, E. Moretti, A. Morselli, L. Pacciani, A. Pellizzoni, F. Perotti, G. Piano, P. Picozza, M. Pilia, G. Porrovecchio, G. Pucella, M. Prest, M. Rapisarda, A. Rappoldi, A. Rubini, P. Soffitta, M. Trifoglio, A. Trois, E. Val- 
lazza, V. Vittorini, A. Zambra, D. Zanello, P. Santolamazza, P. Giommi, S. Colafrancesco, L. A. Antonelli, L. Salotti, Detection of Gamma-Ray Emission from the Eta-Carinae Region, ApJ 698 (2009) L142-L146.

[75] HESS Collaboration, A. Abramowski, F. Acero, F. Aharonian, A. G. Akhperjanian, G. Anton, et al, HESS observations of the Carina nebula and its enigmatic colliding wind binary Eta Carinae, MNRAS 424 (2012) 128-135.

[76] E. Aliu, H. Anderhub, L. A. Antonelli, P. Antoranz, M. Backes, C. Baixeras, J. A. Barrio, H. Bartko, D. Bastieri, J. K. Becker, W. Bednarek, K. Berger, E. Bernardini, C. Bigongiari, A. Biland, R. K. Bock, G. Bonnoli, P. Bordas, V. Bosch-Ramon, T. Bretz, I. Britvitch, M. Camara, E. Carmona, A. Chilingarian, S. Commichau, J. L. Contreras, J. Cortina, M. T. Costado, S. Covino, V. Curtef, F. Dazzi, A. De Angelis, E. De Cea del Pozo, R. de los Reyes, B. De Lotto, M. De Maria, F. De Sabata, C. Delgado Mendez, A. Dominguez, D. Dorner, M. Doro, M. Errando, M. Fagiolini, D. Ferenc, E. Fernández, R. Firpo, M. V. Fonseca, L. Font, N. Galante, R. J. García López, M. Garczarczyk, M. Gaug, F. Goebel, M. Hayashida, A. Herrero, D. Höhne, J. Hose, C. C. Hsu, S. Huber, T. Jogler, D. Kranich, A. La Barbera, A. Laille, E. Leonardo, E. Lindfors, S. Lombardi, F. Longo, M. López, E. Lorenz, P. Majumdar, G. Maneva, N. Mankuzhiyil, K. Mannheim, L. Maraschi, M. Mariotti, M. Martínez, D. Mazin, M. Meucci, M. Meyer, J. M. Miranda, R. Mirzoyan, M. Moles, A. Moralejo, D. Nieto, K. Nilsson, J. Ninkovic, E. Oña-Wilhelmi, N. Otte, I. Oya, R. Paoletti, J. M. Paredes, M. Pasanen, D. Pascoli, F. Pauss, R. G. Pegna, M. A. Perez-Torres, M. Persic, L. Peruzzo, A. Piccioli, F. Prada, E. Prandini, N. Puchades, A. Raymers, W. Rhode, M. Ribó, J. Rico, M. Rissi, A. Robert, S. Rügamer, A. Saggion, T. Y. Saito, M. Salvati, M. SanchezConde, P. Sartori, K. Satalecka, V. Scalzotto, V. Scapin, T. Schweizer, M. Shayduk, K. Shinozaki, S. N. Shore, N. Sidro, A. Sierpowska-Bartosik, A. Sillanpää, D. Sobczynska, F. Spanier, A. Stamerra, L. S. Stark, L. Takalo, F. Tavecchio, P. Temnikov, D. Tescaro, M. Teshima, M. Tluczykont, D. F. Torres, N. Turini, H. Vankov, A. Venturini, V. Vitale, R. M. Wagner, W. Wittek, V. Zabalza, F. Zandanel, R. Zanin, J. Zapatero, First Bounds on the High-Energy Emission from Isolated Wolf-Rayet Binary Systems, ApJ 685 (2008) L71L74.

[77] A. A. Abdo, M. Ackermann, M. Ajello, W. B. Atwood, L. Baldini, J. Ballet, G. Barbiellini, D. Bastieri, K. Bechtol, R. Bellazzini, et al., Gamma-Ray Emission Concurrent with the Nova in the Symbiotic Binary V407 Cygni, Science 329 (2010) 817821.

[78] E. J. A. Meurs, E. P. J. van den Heuvel, The number of evolved early-type close binaries in the Galaxy, A\&A 226 (1989) 88107.

[79] S. F. Portegies Zwart, F. Verbunt, Population synthesis of highmass binaries., A\&A 309 (1996) 179-196.

[80] S. F. Portegies Zwart, L. R. Yungelson, Formation and evolution of binary neutron stars, A\&A 332 (1998) 173-188.

[81] A. M. Atoyan, F. A. Aharonian, Modelling of the non-thermal flares in the Galactic microquasar GRS 1915+105, MNRAS 302 (1999) 253-276

[82] S. Corbel, G. Dubus, J. A. Tomsick, A. Szostek, R. H. D. Corbet, J. C. A. Miller-Jones, J. L. Richards, G. Pooley, S. Trushkin, R. Dubois, A. B. Hill, M. Kerr, W. MaxMoerbeck, A. C. S. Readhead, A. Bodaghee, V. Tudose, D. Parent, J. Wilms, K. Pottschmidt, A giant radio flare from Cygnus $\mathrm{X}-3$ with associated $\gamma$-ray emission, MNRAS 421 (2012) 2947-2955.

[83] A. Sierpowska-Bartosik, D. F. Torres, GLAST Testing of a Pul- sar Model Matching H.E.S.S. Observations of LS 5039, ApJ 674 (2008) L89-L92.

[84] V. A. Acciari, E. Aliu, T. Arlen, T. Aune, M. Beilicke, W. Benbow, S. M. Bradbury, J. H. Buckley, V. Bugaev, K. Byrum, A. Cannon, A. Cesarini, L. Ciupik, E. Collins-Hughes, M. P. Connolly, W. Cui, R. Dickherber, C. Duke, M. Errando, A. Falcone, J. P. Finley, G. Finnegan, L. Fortson, A. Furniss, N. Galante, D. Gall, G. H. Gillanders, S. Godambe, S. Griffin, J. Grube, R. Guenette, G. Gyuk, D. Hanna, J. Holder, G. Hughes, C. M. Hui, T. B. Humensky, P. Kaaret, N. Karlsson, M. Kertzman, D. Kieda, H. Krawczynski, F. Krennrich, M. J. Lang, S. LeBohec, G. Maier, P. Majumdar, S. McArthur, A. McCann, P. Moriarty, R. Mukherjee, R. A. Ong, M. Orr, A. N. Otte, N. Park, J. S. Perkins, M. Pohl, H. Prokoph, J. Quinn, K. Ragan, L. C. Reyes, P. T. Reynolds, E. Roache, H. J. Rose, J. Ruppel, D. B. Saxon, M. Schroedter, G. H. Sembroski, G. D. Senturk, A. W. Smith, D. Staszak, G. Tešić, M. Theiling, S. Thibadeau, K. Tsurusaki, A. Varlotta, V. V. Vassiliev, S. Vincent, M. Vivier, S. P. Wakely, J. E. Ward, T. C. Weekes, A. Weinstein, T. Weisgarber, D. A. Williams, B. Zitzer, VERITAS Observations of the TeV Binary LS I +61 303 During 2008-2010, ApJ 738 (2011) 3-+.

[85] V. A. Acciari, E. Aliu, T. Arlen, M. Bautista, M. Beilicke, W. Benbow, M. Böttcher, S. M. Bradbury, V. Bugaev, Y. Butt, Y. Butt, K. Byrum, A. Cannon, A. Cesarini, Y. C. Chow, L. Ciupik, P. Cogan, P. Colin, W. Cui, M. Daniel, R. Dickherber, T. Ergin, A. Falcone, S. J. Fegan, J. P. Finley, P. Fortin, L. Fortson, A. Furniss, D. Gall, G. H. Gillanders, J. Grube, R. Guenette, G. Gyuk, D. Hanna, E. Hays, J. Holder, D. Horan, C. M. Hui, T. B. Humensky, P. Kaaret, N. Karlsson, D. Kieda, J. Kildea, A. Konopelko, H. Krawczynski, F. Krennrich, M. J. Lang, S. LeBohec, G. Maier, A. McCann, M. McCutcheon, J. Millis, P. Moriarty, R. Mukherjee, T. Nagai, R. A. Ong, A. N. Otte, D. Pandel, J. S. Perkins, J. S. Perkins, M. Pohl, J. Quinn, K. Ragan, L. C. Reyes, P. T. Reynolds, E. Roache, H. Joachim Rose, M. Schroedter, G. H. Sembroski, A. W. Smith, D. Steele, M. Stroh, S. Swordy, M. Theiling, J. A. Toner, A. Varlotta, V. V. Vassiliev, R. G. Wagner, S. P. Wakely, J. E. Ward, T. C. Weekes, A. Weinstein, R. J. White, D. A. Williams, S. Wissel, M. Wood, B. Zitzer, Multiwavelength Observations of LS I +61 303 with Veritas, Swift, and RXTE, ApJ 700 (2009) 1034-1041.

[86] N. Rea, D. F. Torres, M. van der Klis, P. G. Jonker, M. Méndez, A. Sierpowska-Bartosik, Deep Chandra observations of TeV binaries - I. LSI+61303, MNRAS 405 (2010) 2206-2214.

[87] L. Sidoli, A. Pellizzoni, S. Vercellone, M. Moroni, S. Mereghetti, M. Tavani, XMM-Newton observation of a spectral state transition in the peculiar radio/X-ray/ $\gamma$-ray source LS I +61 303, A\&A 459 (2006) 901-907.

[88] V. Bosch-Ramon, G. E. Romero, J. M. Paredes, A broadband leptonic model for gamma-ray emitting microquasars, A\&A 447 (2006) 263-276.

[89] A. A. Zdziarski, A. Neronov, M. Chernyakova, A compact pulsar wind nebula model of the $\gamma$-ray-loud binary LS I +61303 , MNRAS 403 (2010) 1873-1886.

[90] T. Jogler, Detailed study of the binary system LS I +61o303 in VHE gamma-rays with the MAGIC telescope, Ph.D. thesis, Technische Universität München, 2009.

[91] V. Zabalza, J. M. Paredes, V. Bosch-Ramon, On the origin of correlated X-ray/VHE emission from LS I +61 303, A\&A 527 (2011) A9+.

[92] R. A. Edelson, J. H. Krolik, The discrete correlation function A new method for analyzing unevenly sampled variability data, ApJ 333 (1988) 646-659.

[93] T. Alexander, Is AGN Variability Correlated with Other AGN 
Properties? ZDCF Analysis of Small Samples of Sparse Light Curves, in: D. Maoz, A. Sternberg, \& E. M. Leibowitz (Ed.), Astronomical Time Series, volume 218 of Astrophysics and Space Science Library, pp. 163-+.

[94] S. Safi-Harb, H. Oegelman, ROSAT and ASCA Observations of W50 Associated with the Peculiar Source SS 433, ApJ 483 (1997) 868.

[95] C. R. Kaiser, K. F. Gunn, C. Brocksopp, J. L. Sokoloski, Revision of the Properties of the GRS 1915+105 Jets: Clues from the Large-Scale Structure, ApJ 612 (2004) 332-341.

[96] A. A. Zdziarski, M. Gierliński, A. R. Rao, S. V. Vadawale, J. Mikołajewska, GRS 1915+105: the distance, radiative processes and energy-dependent variability, MNRAS 360 (2005) 825-838.

[97] E. Gallo, R. Fender, C. Kaiser, D. Russell, R. Morganti, T. Oosterloo, S. Heinz, A dark jet dominates the power output of the stellar black hole Cygnus X-1, Nature 436 (2005) 819-821.

[98] C. R. Kaiser, P. Alexander, A self-similar model for extragalactic radio sources, MNRAS 286 (1997) 215-222.

[99] HESS Collaboration, A. Abramowski, F. Acero, F. Aharonian, A. G. Akhperjanian, G. Anton, et al, H.E.S.S. observations of the Carina nebula and its enigmatic colliding wind binary Eta Carinae, ArXiv e-prints (2012).

[100] Y. Sugawara, Y. Maeda, Y. Tsuboi, K. Hamaguchi, M. Corcoran, A. Pollock, A. Moffat, P. Williams, S. Dougherty, J. Pittard, The variable X-ray spectrum of the Wolf-Rayet binary WR140 with Suzaku, Bulletin de la Societe Royale des Sciences de Liege 80 (2011) 724-728. 